\title{
Theoretical Studies of Quadruply Ionized Radon (Rn V) for Energetically Low Lying Levels - Supplement
}

\author{
B. Karaçoban Usta ${ }^{a, *}$ And S. Eser ${ }^{b}$ \\ ${ }^{a}$ Department of Fundamental Science in Engineering, Sakarya University of Applied Science, \\ 54050, Sakarya, Turkey \\ ${ }^{b}$ Department of Physics, Sakarya University, 54050, Sakarya, Turkey
}

Received: 22.10.2020 \& Accepted: 30.01.2021

Doi: 10.12693/APhysPolA.139.132.S1

*e-mail: bkaracoban@subu.edu.tr topics: HFR method, MCDF method, relativistic corrections, wavelengths

TABLE SI

New energies and Landé $g$-factors for Rn V. ${ }^{*}$ shows the level that are not the dominant component of the eigenvector.

\begin{tabular}{c|l|c|c|c}
\hline \hline \multicolumn{2}{c|}{ Levels } & $E\left[\mathrm{~cm}^{-1}\right]$ & $g$-factor \\
\hline Conf. & Term & $J$ & HFR & HFR \\
\hline $6 s^{2} 6 p\left({ }^{2} P\right) 9 p$ & ${ }^{3} D$ & 1 & 344649.99 & 0.669 \\
$6 s^{2} 6 p\left({ }^{2} P\right) 9 p$ & ${ }^{3} P$ & 0 & 345503.71 & 0.00 \\
$6 s^{2} 6 p\left({ }^{2} P\right) 9 p$ & ${ }^{3} D$ & 2 & 347955.00 & 1.166 \\
$6 s^{2} 6 p\left({ }^{2} P\right) 9 p$ & ${ }^{3} S^{*}$ & 1 & 348003.59 & 1.486 \\
$6 s^{2} 6 p\left({ }^{2} P\right) 9 p$ & ${ }^{3} P$ & 1 & 389542.47 & 1.384 \\
$6 s^{2} 6 p\left({ }^{2} P\right) 9 p$ & ${ }^{1} D^{*}$ & 2 & 389671.32 & 1.182 \\
$6 s^{2} 6 p\left({ }^{2} P\right) 9 p$ & ${ }^{3} D$ & 3 & 392485.60 & 1.329 \\
$6 s^{2} 6 p\left({ }^{2} P\right) 9 p$ & ${ }^{1} P^{*}$ & 1 & 392509.80 & 1.458 \\
$6 s^{2} 6 p\left({ }^{2} P\right) 9 p$ & ${ }^{3} P$ & 2 & 392922.89 & 1.277 \\
$6 s^{2} 6 p\left({ }^{2} P\right) 9 p$ & ${ }^{1} S$ & 0 & 394304.59 & 0.00 \\
\hline $6 s^{2} 6 p\left({ }^{2} P\right) 10 p$ & ${ }^{3} D$ & 1 & 368102.80 & 0.665 \\
$6 s^{2} 6 p\left({ }^{2} P\right) 10 p$ & ${ }^{3} P$ & 0 & 368751.72 & 0.00 \\
$6 s^{2} 6 p\left({ }^{2} P\right) 10 p$ & ${ }^{3} S^{*}$ & 1 & 370173.10 & 1.497 \\
$6 s^{2} 6 p\left({ }^{2} P\right) 10 p$ & ${ }^{3} D$ & 2 & 370241.46 & 1.168 \\
$6 s^{2} 6 p\left({ }^{2} P\right) 10 p$ & ${ }^{3} P$ & 1 & 412927.28 & 1.390 \\
$6 s^{2} 6 p\left({ }^{2} P\right) 10 p$ & ${ }^{1} D^{*}$ & 2 & 413040.08 & 1.206 \\
$6 s^{2} 6 p\left({ }^{2} P\right) 10 p$ & ${ }^{3} D$ & 3 & 414718.02 & 1.334 \\
$6 s^{2} 6 p\left({ }^{2} P\right) 10 p$ & ${ }^{1} P$ & 1 & 414761.09 & 1.449 \\
$6 s^{2} 6 p\left({ }^{2} P\right) 10 p$ & ${ }^{3} P$ & 2 & 414971.10 & 1.292 \\
$6 s^{2} 6 p\left({ }^{2} P\right) 10 p$ & ${ }^{1} S$ & 0 & 415847.39 & 0.00 \\
\hline $6 s^{2} 6 p\left({ }^{2} P\right) 6 f$ & ${ }^{3} G$ & 3 & 291043.32 & 0.829 \\
$6 s^{2} 6 p\left({ }^{2} P\right) 6 f$ & ${ }^{3} F$ & 2 & 292136.69 & 0.874 \\
$6 s^{2} 6 p\left({ }^{2} P\right) 6 f$ & ${ }^{3} D$ & 3 & 292136.89 & 1.194 \\
$6 s^{2} 6 p\left({ }^{2} P\right) 6 f$ & ${ }^{3} G$ & 4 & 292258.48 & 1.090 \\
$6 s^{2} 6 p\left({ }^{2} P\right) 6 f$ & ${ }^{1} F$ & 3 & 333794.58 & 0.960 \\
$6 s^{2} 6 p\left({ }^{2} P\right) 6 f$ & ${ }^{3} F$ & 4 & 334469.72 & 1.184 \\
$6 s^{2} 6 p\left({ }^{2} P\right) 6 f$ & ${ }^{3} D^{*}$ & 2 & 334940.67 & 0.888 \\
$6 s^{2} 6 p\left({ }^{2} P\right) 6 f$ & ${ }^{3} F^{*}$ & 3 & 335235.40 & 1.185 \\
& & & \\
& & & \\
\hline
\end{tabular}

TABLE SI (cont.)

\begin{tabular}{|c|c|c|c|c|}
\hline \multicolumn{3}{|c|}{ Levels } & $E\left[\mathrm{~cm}^{-1}\right]$ & $g$-factor \\
\hline Conf. & Term & $J$ & HFR & HFR \\
\hline $6 s^{2} 6 p\left({ }^{2} P\right) 6 f$ & ${ }^{3} G$ & 5 & 336079.48 & 1.200 \\
\hline $6 s^{2} 6 p\left({ }^{2} P\right) 6 f$ & ${ }^{1} G$ & 4 & 336392.31 & 1.028 \\
\hline $6 s^{2} 6 p\left({ }^{2} P\right) 6 f$ & ${ }^{3} D$ & 1 & 336856.48 & 0.499 \\
\hline $6 s^{2} 6 p\left({ }^{2} P\right) 6 f$ & ${ }^{1} D$ & 2 & 338434.09 & 0.966 \\
\hline $6 s^{2} 6 p\left({ }^{2} P\right) 7 f$ & ${ }^{3} G$ & 3 & 337482.98 & 0.826 \\
\hline $6 s^{2} 6 p\left({ }^{2} P\right) 7 f$ & ${ }^{3} D^{*}$ & 2 & 337579.05 & 0.993 \\
\hline $6 s^{2} 6 p\left({ }^{2} P\right) 7 f$ & ${ }^{3} D$ & 3 & 338077.12 & 1.201 \\
\hline $6 s^{2} 6 p\left({ }^{2} P\right) 7 f$ & ${ }^{3} G$ & 4 & 338174.66 & 1.084 \\
\hline $6 s^{2} 6 p\left({ }^{2} P\right) 7 f$ & ${ }^{3} F$ & 3 & 381249.02 & 0.962 \\
\hline $6 s^{2} 6 p\left({ }^{2} P\right) 7 f$ & ${ }^{3} F$ & 4 & 381573.17 & 1.183 \\
\hline $6 s^{2} 6 p\left({ }^{2} P\right) 7 f$ & ${ }^{3} F$ & 2 & 381928.57 & 0.892 \\
\hline $6 s^{2} 6 p\left({ }^{2} P\right) 7 f$ & ${ }^{1} F^{*}$ & 3 & 382078.61 & 1.177 \\
\hline $6 s^{2} 6 p\left({ }^{2} P\right) 7 f$ & ${ }^{3} G$ & 5 & 382330.70 & 1.200 \\
\hline $6 s^{2} 6 p\left({ }^{2} P\right) 7 f$ & ${ }^{1} G$ & 4 & 382427.29 & 1.028 \\
\hline $6 s^{2} 6 p\left({ }^{2} P\right) 7 f$ & ${ }^{3} D$ & 1 & 382637.29 & 0.499 \\
\hline $6 s^{2} 6 p\left({ }^{2} P\right) 7 f$ & ${ }^{1} D$ & 2 & 383209.10 & 1.066 \\
\hline $6 s^{2} 6 p\left({ }^{2} P\right) 8 f$ & ${ }^{3} G$ & 3 & 363611.38 & 0.826 \\
\hline $6 s^{2} 6 p\left({ }^{2} P\right) 8 f$ & ${ }^{3} F$ & 2 & 363791.10 & 0.885 \\
\hline $6 s^{2} 6 p\left({ }^{2} P\right) 8 f$ & ${ }^{3} D$ & 3 & 363871.45 & 1.201 \\
\hline $6 s^{2} 6 p\left({ }^{2} P\right) 8 f$ & ${ }^{3} G$ & 4 & 363904.15 & 1.085 \\
\hline $6 s^{2} 6 p\left({ }^{2} P\right) 8 f$ & ${ }^{3} F$ & 3 & 407822.51 & 0.969 \\
\hline $6 s^{2} 6 p\left({ }^{2} P\right) 8 f$ & ${ }^{3} F$ & 4 & 408011.28 & 1.189 \\
\hline $6 s^{2} 6 p\left({ }^{2} P\right) 8 f$ & ${ }^{3} D$ & 2 & 408169.52 & 0.881 \\
\hline $6 s^{2} 6 p\left({ }^{2} P\right) 8 f$ & ${ }^{1} F^{*}$ & 3 & 408273.37 & 1.172 \\
\hline $6 s^{2} 6 p\left({ }^{2} P\right) 8 f$ & ${ }^{3} G$ & 5 & 408475.50 & 1.200 \\
\hline $6 s^{2} 6 p\left({ }^{2} P\right) 8 f$ & ${ }^{1} G$ & 4 & 408502.98 & 1.027 \\
\hline $6 s^{2} 6 p\left({ }^{2} P\right) 8 f$ & ${ }^{3} D$ & 1 & 408616.78 & 0.499 \\
\hline $6 s^{2} 6 p\left({ }^{2} P\right) 8 f$ & ${ }^{1} D$ & 2 & 408934.82 & 1.069 \\
\hline $6 s^{2} 6 p\left({ }^{2} P\right) 9 f$ & ${ }^{3} G$ & 3 & 379902.82 & 0.836 \\
\hline $6 s^{2} 6 p\left({ }^{2} P\right) 9 f$ & ${ }^{3} F$ & 2 & 379914.69 & 0.884 \\
\hline $6 s^{2} 6 p\left({ }^{2} P\right) 9 f$ & ${ }^{3} D$ & 3 & 380015.68 & 1.195 \\
\hline
\end{tabular}


TABLE SI (cont.)

TABLE SI (cont.)

\begin{tabular}{|c|c|c|c|c|}
\hline \multicolumn{3}{|c|}{ Levels } & $E\left[\mathrm{~cm}^{-1}\right]$ & $g$-factor \\
\hline Conf. & Term & $J$ & HFR & HFR \\
\hline $6 s^{2} 6 p\left({ }^{2} P\right) 9 f$ & ${ }^{3} G$ & 4 & 380074.99 & 1.087 \\
\hline $6 s^{2} 6 p\left({ }^{2} P\right) 9 f$ & ${ }^{3} F$ & 3 & 424353.07 & 0.970 \\
\hline $6 s^{2} 6 p\left({ }^{2} P\right) 9 f$ & ${ }^{3} F$ & 4 & 424472.53 & 1.189 \\
\hline $6 s^{2} 6 p\left({ }^{2} P\right) 9 f$ & ${ }^{3} D^{*}$ & 2 & 424573.46 & 0.881 \\
\hline $6 s^{2} 6 p\left({ }^{2} P\right) 9 f$ & ${ }^{1} F^{*}$ & 3 & 424635.80 & 1.171 \\
\hline $6 s^{2} 6 p\left({ }^{2} P\right) 9 f$ & ${ }^{3} G$ & 5 & 424759.98 & 1.200 \\
\hline $6 s^{2} 6 p\left({ }^{2} P\right) 9 f$ & ${ }^{1} G$ & 4 & 424772.12 & 1.027 \\
\hline $6 s^{2} 6 p\left({ }^{2} P\right) 9 f$ & ${ }^{3} D$ & 1 & 424848.30 & 0.499 \\
\hline $6 s^{2} 6 p\left({ }^{2} P\right) 9 f$ & ${ }^{1} D$ & 2 & 425057.69 & 1.066 \\
\hline $6 s^{2} 6 p\left({ }^{2} P\right) 10 f$ & ${ }^{3} G$ & 3 & 390837.79 & 0.837 \\
\hline $6 s^{2} 6 p\left({ }^{2} P\right) 10 f$ & ${ }^{3} D$ & 3 & 390898.99 & 1.195 \\
\hline $6 s^{2} 6 p\left({ }^{2} P\right) 10 f$ & ${ }^{3} F$ & 2 & 390956.70 & 0.924 \\
\hline $6 s^{2} 6 p\left({ }^{2} P\right) 10 f$ & ${ }^{3} G$ & 4 & 390967.00 & 1.084 \\
\hline $6 s^{2} 6 p\left({ }^{2} P\right) 10 f$ & ${ }^{3} F$ & 3 & 435365.22 & 0.970 \\
\hline $6 s^{2} 6 p\left({ }^{2} P\right) 10 f$ & ${ }^{3} F$ & 4 & 435445.66 & 1.189 \\
\hline $6 s^{2} 6 p\left({ }^{2} P\right) 10 f$ & ${ }^{3} D^{*}$ & 2 & 435503.59 & 0.878 \\
\hline $6 s^{2} 6 p\left({ }^{2} P\right) 10 f$ & ${ }^{1} F^{*}$ & 3 & 435549.02 & 1.169 \\
\hline $6 s^{2} 6 p\left({ }^{2} P\right) 10 f$ & ${ }^{3} G$ & 5 & 435635.49 & 1.200 \\
\hline $6 s^{2} 6 p\left({ }^{2} P\right) 10 f$ & ${ }^{1} G$ & 4 & 435643.38 & 1.027 \\
\hline $6 s^{2} 6 p\left({ }^{2} P\right) 10 f$ & ${ }^{3} D$ & 1 & 435694.19 & 0.499 \\
\hline $6 s^{2} 6 p\left({ }^{2} P\right) 10 f$ & ${ }^{1} D$ & 2 & 435834.90 & 1.068 \\
\hline $6 s^{2} 6 p\left({ }^{2} P\right) 6 h$ & ${ }^{3} I$ & 5 & 351958.00 & 0.885 \\
\hline $6 s^{2} 6 p\left({ }^{2} P\right) 6 h$ & ${ }^{3} I$ & 6 & 351976.38 & 1.057 \\
\hline $6 s^{2} 6 p\left({ }^{2} P\right) 6 h$ & ${ }^{3} G$ & 5 & 352005.63 & 1.126 \\
\hline $6 s^{2} 6 p\left({ }^{2} P\right) 6 h$ & ${ }^{1} G^{*}$ & 4 & 352011.87 & 0.931 \\
\hline $6 s^{2} 6 p\left({ }^{2} P\right) 6 h$ & ${ }^{1} H$ & 5 & 396362.11 & 0.963 \\
\hline $6 s^{2} 6 p\left({ }^{2} P\right) 6 h$ & ${ }^{3} H$ & 6 & 396374.08 & 1.123 \\
\hline $6 s^{2} 6 p\left({ }^{2} P\right) 6 h$ & ${ }^{3} H^{*}$ & 5 & 396541.67 & 1.093 \\
\hline $6 s^{2} 6 p\left({ }^{2} P\right) 6 h$ & ${ }^{3} H$ & 4 & 396546.44 & 0.892 \\
\hline $6 s^{2} 6 p\left({ }^{2} P\right) 6 h$ & ${ }^{3} I$ & 7 & 396898.68 & 1.143 \\
\hline $6 s^{2} 6 p\left({ }^{2} P\right) 6 h$ & ${ }^{1} I$ & 6 & 396916.27 & 1.011 \\
\hline $6 s^{2} 6 p\left({ }^{2} P\right) 6 h$ & ${ }^{3} G$ & 3 & 397068.98 & 0.749 \\
\hline $6 s^{2} 6 p\left({ }^{2} P\right) 6 h$ & ${ }^{3} G$ & 4 & 397086.43 & 1.027 \\
\hline $6 s^{2} 6 p\left({ }^{2} P\right) 7 h$ & ${ }^{3} I$ & 5 & 372477.96 & 0.885 \\
\hline $6 s^{2} 6 p\left({ }^{2} P\right) 7 h$ & ${ }^{3} G$ & 5 & 372490.32 & 1.126 \\
\hline $6 s^{2} 6 p\left({ }^{2} P\right) 7 h$ & ${ }^{3} I$ & 6 & 372495.77 & 1.056 \\
\hline $6 s^{2} 6 p\left({ }^{2} P\right) 7 h$ & ${ }^{1} G^{*}$ & 4 & 372497.62 & 0.932 \\
\hline $6 s^{2} 6 p\left({ }^{2} P\right) 7 h$ & ${ }^{1} H$ & 5 & 417005.11 & 0.964 \\
\hline $6 s^{2} 6 p\left({ }^{2} P\right) 7 h$ & ${ }^{3} H$ & 6 & 417014.99 & 1.124 \\
\hline $6 s^{2} 6 p\left({ }^{2} P\right) 7 h$ & ${ }^{3} H^{*}$ & 5 & 417114.37 & 1.092 \\
\hline $6 s^{2} 6 p\left({ }^{2} P\right) 7 h$ & ${ }^{3} H$ & 4 & 417120.30 & 0.891 \\
\hline $6 s^{2} 6 p\left({ }^{2} P\right) 7 h$ & ${ }^{3} I$ & 7 & 417335.42 & 1.143 \\
\hline $6 s^{2} 6 p\left({ }^{2} P\right) 7 h$ & ${ }^{1} I$ & 6 & 417356.68 & 1.011 \\
\hline $6 s^{2} 6 p\left({ }^{2} P\right) 7 h$ & ${ }^{3} G$ & 3 & 417446.49 & 0.749 \\
\hline $6 s^{2} 6 p\left({ }^{2} P\right) 7 h$ & ${ }^{3} G$ & 4 & 417463.59 & 1.027 \\
\hline $6 s^{2} 6 p\left({ }^{2} P\right) 8 h$ & ${ }^{3} I$ & 5 & 385726.39 & 0.884 \\
\hline $6 s^{2} 6 p\left({ }^{2} P\right) 8 h$ & ${ }^{3} I$ & 6 & 385740.75 & 1.056 \\
\hline $6 s^{2} 6 p\left({ }^{2} P\right) 8 h$ & ${ }^{3} G$ & 5 & 385757.91 & 1.127 \\
\hline $6 s^{2} 6 p\left({ }^{2} P\right) 8 h$ & ${ }^{1} G^{*}$ & 4 & 385768.22 & 0.933 \\
\hline
\end{tabular}

\begin{tabular}{|c|c|c|c|c|}
\hline \multicolumn{3}{|c|}{ Levels } & $E\left[\mathrm{~cm}^{-1}\right]$ & $g$-factor \\
\hline Conf. & Term & $J$ & HFR & HFR \\
\hline $6 s^{2} 6 p\left({ }^{2} P\right) 8 h$ & ${ }^{1} H$ & 5 & 430326.61 & 0.964 \\
\hline $6 s^{2} 6 p\left({ }^{2} P\right) 8 h$ & ${ }^{3} H$ & 6 & 430334.10 & 1.124 \\
\hline $6 s^{2} 6 p\left({ }^{2} P\right) 8 h$ & ${ }^{3} H^{*}$ & 5 & 430398.09 & 1.092 \\
\hline $6 s^{2} 6 p\left({ }^{2} P\right) 8 h$ & ${ }^{3} H$ & 4 & 430403.67 & 0.891 \\
\hline $6 s^{2} 6 p\left({ }^{2} P\right) 8 h$ & ${ }^{3} I$ & 7 & 430542.60 & 1.143 \\
\hline $6 s^{2} 6 p\left({ }^{2} P\right) 8 h$ & ${ }^{1} I$ & 6 & 430561.79 & 1.011 \\
\hline $6 s^{2} 6 p\left({ }^{2} P\right) 8 h$ & ${ }^{3} G$ & 3 & 430619.09 & 0.749 \\
\hline $6 s^{2} 6 p\left({ }^{2} P\right) 8 h$ & ${ }^{3} G$ & 4 & 430633.41 & 1.026 \\
\hline $6 s^{2} 6 p\left({ }^{2} P\right) 9 h$ & ${ }^{3} I$ & 5 & 394821.41 & 0.886 \\
\hline $6 s^{2} 6 p\left({ }^{2} P\right) 9 h$ & ${ }^{3} G$ & 5 & 394828.60 & 1.126 \\
\hline $6 s^{2} 6 p\left({ }^{2} P\right) 9 h$ & ${ }^{3} I$ & 6 & 394832.00 & 1.056 \\
\hline $6 s^{2} 6 p\left({ }^{2} P\right) 9 h$ & ${ }^{1} G^{*}$ & 4 & 394834.27 & 0.932 \\
\hline $6 s^{2} 6 p\left({ }^{2} P\right) 9 h$ & ${ }^{1} H$ & 5 & 439471.50 & 0.964 \\
\hline $6 s^{2} 6 p\left({ }^{2} P\right) 9 h$ & ${ }^{3} H$ & 6 & 439477.15 & 1.124 \\
\hline $6 s^{2} 6 p\left({ }^{2} P\right) 9 h$ & ${ }^{3} H^{*}$ & 5 & 439520.70 & 1.092 \\
\hline $6 s^{2} 6 p\left({ }^{2} P\right) 9 h$ & ${ }^{3} H$ & 4 & 439525.45 & 0.891 \\
\hline $6 s^{2} 6 p\left({ }^{2} P\right) 9 h$ & ${ }^{3} I$ & 7 & 439620.21 & 1.143 \\
\hline $6 s^{2} 6 p\left({ }^{2} P\right) 9 h$ & ${ }^{1} I$ & 6 & 439636.20 & 1.011 \\
\hline $6 s^{2} 6 p\left({ }^{2} P\right) 9 h$ & ${ }^{3} G$ & 3 & 439675.24 & 0.749 \\
\hline $6 s^{2} 6 p\left({ }^{2} P\right) 9 h$ & ${ }^{3} G$ & 4 & 439687.00 & 1.026 \\
\hline $6 s^{2} 6 p\left({ }^{2} P\right) 10 h$ & ${ }^{3} I$ & 5 & 401327.86 & 0.884 \\
\hline $6 s^{2} 6 p\left({ }^{2} P\right) 10 h$ & ${ }^{3} G$ & 5 & 401335.18 & 1.127 \\
\hline $6 s^{2} 6 p\left({ }^{2} P\right) 10 h$ & ${ }^{3} I$ & 6 & 401336.92 & 1.056 \\
\hline $6 s^{2} 6 p\left({ }^{2} P\right) 10 h$ & ${ }^{1} G^{*}$ & 4 & 401340.66 & 0.933 \\
\hline $6 s^{2} 6 p\left({ }^{2} P\right) 10 h$ & ${ }^{1} H$ & 5 & 445999.87 & 0.964 \\
\hline $6 s^{2} 6 p\left({ }^{2} P\right) 10 h$ & ${ }^{3} H$ & 6 & 446003.93 & 1.124 \\
\hline $6 s^{2} 6 p\left({ }^{2} P\right) 10 h$ & ${ }^{3} H^{*}$ & 5 & 446034.52 & 1.092 \\
\hline $6 s^{2} 6 p\left({ }^{2} P\right) 10 h$ & ${ }^{3} H$ & 4 & 446038.42 & 0.891 \\
\hline $6 s^{2} 6 p\left({ }^{2} P\right) 10 h$ & ${ }^{3} I$ & 7 & 446106.08 & 1.143 \\
\hline $6 s^{2} 6 p\left({ }^{2} P\right) 10 h$ & ${ }^{1} I$ & 6 & 446119.41 & 1.011 \\
\hline $6 s^{2} 6 p\left({ }^{2} P\right) 10 h$ & ${ }^{3} G$ & 3 & 446147.64 & 0.749 \\
\hline $6 s^{2} 6 p\left({ }^{2} P\right) 10 h$ & ${ }^{3} G$ & 4 & 446156.96 & 1.026 \\
\hline $6 s^{2} 6 p\left({ }^{2} P\right) 9 s$ & ${ }^{3} P^{o}$ & 0 & 335813.99 & 0.00 \\
\hline $6 s^{2} 6 p\left({ }^{2} P\right) 9 s$ & ${ }^{3} P^{o}$ & 1 & 336667.58 & 1.154 \\
\hline $6 s^{2} 6 p\left({ }^{2} P\right) 9 s$ & ${ }^{3} P^{o}$ & 2 & 380449.90 & 1.458 \\
\hline $6 s^{2} 6 p\left({ }^{2} P\right) 9 s$ & ${ }^{1} P^{o}$ & 1 & 380535.28 & 1.210 \\
\hline $6 \mathrm{~s}^{2} 6 \mathrm{p}\left({ }^{2} \mathrm{P}\right) 10 \mathrm{~s}$ & ${ }^{3} P^{o}$ & 0 & 362702.69 & 0.00 \\
\hline $6 \mathrm{~s}^{2} 6 \mathrm{p}\left({ }^{2} \mathrm{P}\right) 10 \mathrm{~s}$ & ${ }^{3} P^{o}$ & 1 & 362827.10 & 1.312 \\
\hline $6 \mathrm{~s}^{2} 6 \mathrm{p}\left({ }^{2} \mathrm{P}\right) 10 \mathrm{~s}$ & ${ }^{3} P^{o}$ & 2 & 407416.90 & 1.442 \\
\hline $6 \mathrm{~s}^{2} 6 \mathrm{p}\left({ }^{2} \mathrm{P}\right) 10 \mathrm{~s}$ & ${ }^{1} P^{o}$ & 1 & 407483.80 & 1.216 \\
\hline $6 s^{2} 6 p\left({ }^{2} P\right) 8 d$ & ${ }^{3} F^{o}$ & 2 & 334173.89 & 0.766 \\
\hline $6 s^{2} 6 p\left({ }^{2} P\right) 8 d$ & ${ }^{3} D^{o}$ & 1 & 335490.22 & 1.026 \\
\hline $6 s^{2} 6 p\left({ }^{2} P\right) 8 d$ & ${ }^{3} P^{o}$ & 2 & 335558.61 & 1.286 \\
\hline $6 s^{2} 6 p\left({ }^{2} P\right) 8 d$ & ${ }^{3} F^{o}$ & 3 & 336217.78 & 1.109 \\
\hline $6 s^{2} 6 p\left({ }^{2} P\right) 8 d$ & ${ }^{1} D^{o}$ & 2 & 378615.60 & 0.987 \\
\hline $6 s^{2} 6 p\left({ }^{2} P\right) 8 d$ & ${ }^{3} D^{o *}$ & 3 & 379010.27 & 1.137 \\
\hline $6 s^{2} 6 p\left({ }^{2} P\right) 9 d$ & ${ }^{3} F^{o}$ & 2 & 362057.30 & 0.742 \\
\hline $6 s^{2} 6 p\left({ }^{2} P\right) 8 d$ & ${ }^{3} P^{o}$ & 1 & 379554.02 & 1.071 \\
\hline $6 s^{2} 6 p\left({ }^{2} P\right) 8 d$ & ${ }^{3} F^{o}$ & 4 & 379566.90 & 1.250 \\
\hline
\end{tabular}


TABLE SI (cont.)

TABLE SI (cont.)

\begin{tabular}{|c|c|c|c|c|}
\hline \multicolumn{3}{|c|}{ Levels } & $E\left[\mathrm{~cm}^{-1}\right]$ & $g$-factor \\
\hline Conf. & Term & $J$ & HFR & HFR \\
\hline $6 s^{2} 6 p\left({ }^{2} P\right) 8 d$ & ${ }^{3} P^{o}$ & 0 & 379760.89 & 0.00 \\
\hline $6 s^{2} 6 p\left({ }^{2} P\right) 8 d$ & ${ }^{3} D^{o *}$ & 2 & 379861.07 & 1.331 \\
\hline $6 s^{2} 6 p\left({ }^{2} P\right) 8 d$ & ${ }^{1} F^{o}$ & 3 & 380841.47 & 1.099 \\
\hline $6 s^{2} 6 p\left({ }^{2} P\right) 8 d$ & ${ }^{1} P^{o}$ & 1 & 381732.82 & 1.031 \\
\hline $6 s^{2} 6 p\left({ }^{2} P\right) 9 d$ & ${ }^{3} D^{o}$ & 1 & 362446.70 & 0.850 \\
\hline $6 s^{2} 6 p\left({ }^{2} P\right) 9 d$ & ${ }^{3} P^{o}$ & 2 & 362658.69 & 1.280 \\
\hline $6 s^{2} 6 p\left({ }^{2} P\right) 9 d$ & ${ }^{3} F^{o}$ & 3 & 362814.78 & 1.112 \\
\hline $6 s^{2} 6 p\left({ }^{2} P\right) 9 d$ & ${ }^{3} D^{o *}$ & 2 & 406470.61 & 0.993 \\
\hline $6 s^{2} 6 p\left({ }^{2} P\right) 9 d$ & ${ }^{3} D^{o}$ & 3 & 406768.96 & 1.193 \\
\hline $6 s^{2} 6 p\left({ }^{2} P\right) 9 d$ & ${ }^{3} P^{o}$ & 1 & 406888.37 & 1.071 \\
\hline $6 s^{2} 6 p\left({ }^{2} P\right) 9 d$ & ${ }^{3} F^{o}$ & 4 & 407041.12 & 1.250 \\
\hline $6 s^{2} 6 p\left({ }^{2} P\right) 9 d$ & ${ }^{1} D^{o *}$ & 2 & 407095.47 & 1.347 \\
\hline $6 s^{2} 6 p\left({ }^{2} P\right) 9 d$ & ${ }^{3} P^{o}$ & 0 & 407105.11 & 0.00 \\
\hline $6 s^{2} 6 p\left({ }^{2} P\right) 9 d$ & ${ }^{1} F^{o}$ & 3 & 407600.64 & 1.111 \\
\hline $6 s^{2} 6 p\left({ }^{2} P\right) 9 d$ & ${ }^{1} P^{o}$ & 1 & 408135.57 & 1.052 \\
\hline $6 s^{2} 6 p\left({ }^{2} P\right) 10 d$ & ${ }^{3} F^{o}$ & 2 & 378903.38 & 0.789 \\
\hline $6 s^{2} 6 p\left({ }^{2} P\right) 10 d$ & ${ }^{3} D^{o}$ & 1 & 379019.91 & 0.859 \\
\hline $6 s^{2} 6 p\left({ }^{2} P\right) 10 d$ & ${ }^{3} P^{o}$ & 2 & 379295.19 & 1.273 \\
\hline $6 s^{2} 6 p\left({ }^{2} P\right) 10 d$ & ${ }^{3} F^{o *}$ & 3 & 379445.72 & 1.179 \\
\hline $6 s^{2} 6 p\left({ }^{2} P\right) 10 d$ & ${ }^{3} D^{o}$ & 2 & 423548.21 & 0.995 \\
\hline $6 s^{2} 6 p\left({ }^{2} P\right) 10 d$ & ${ }^{3} D^{o}$ & 3 & 423733.00 & 1.191 \\
\hline $6 s^{2} 6 p\left({ }^{2} P\right) 10 d$ & ${ }^{3} P^{o}$ & 1 & 423832.67 & 1.097 \\
\hline $6 s^{2} 6 p\left({ }^{2} P\right) 10 d$ & ${ }^{3} F^{o}$ & 4 & 423916.79 & 1.251 \\
\hline $6 s^{2} 6 p\left({ }^{2} P\right) 10 d$ & ${ }^{3} P^{o}$ & 0 & 423941.01 & 0.00 \\
\hline $6 s^{2} 6 p\left({ }^{2} P\right) 10 d$ & ${ }^{1} D^{o *}$ & 2 & 423997.60 & 1.285 \\
\hline $6 s^{2} 6 p\left({ }^{2} P\right) 10 d$ & ${ }^{1} F^{o}$ & 3 & 424245.28 & 1.114 \\
\hline $6 s^{2} 6 p\left({ }^{2} P\right) 10 d$ & ${ }^{1} P^{o}$ & 1 & 424525.79 & 1.074 \\
\hline $6 s^{2} 6 p\left({ }^{2} P\right) 5 g$ & ${ }^{3} H^{o}$ & 4 & 315811.72 & 0.867 \\
\hline $6 s^{2} 6 p\left({ }^{2} P\right) 5 g$ & ${ }^{3} F^{o}$ & 4 & 315985.88 & 1.147 \\
\hline $6 s^{2} 6 p\left({ }^{2} P\right) 5 g$ & ${ }^{3} H^{o}$ & 5 & 316170.71 & 1.071 \\
\hline $6 s^{2} 6 p\left({ }^{2} P\right) 5 g$ & ${ }^{1} F^{o *}$ & 3 & 316303.36 & 0.907 \\
\hline $6 s^{2} 6 p\left({ }^{2} P\right) 5 g$ & ${ }^{1} G^{o}$ & 4 & 359474.58 & 0.957 \\
\hline $6 s^{2} 6 p\left({ }^{2} P\right) 5 g$ & ${ }^{3} G^{o}$ & 5 & 359639.62 & 1.147 \\
\hline $6 s^{2} 6 p\left({ }^{2} P\right) 5 g$ & ${ }^{3} G^{o *}$ & 4 & 360212.19 & 1.129 \\
\hline $6 s^{2} 6 p\left({ }^{2} P\right) 5 g$ & ${ }^{3} F^{o *}$ & 3 & 360389.68 & 0.892 \\
\hline $6 s^{2} 6 p\left({ }^{2} P\right) 5 g$ & ${ }^{3} H^{o}$ & 6 & 360866.38 & 1.167 \\
\hline $6 s^{2} 6 p\left({ }^{2} P\right) 5 g$ & ${ }^{1} H^{o}$ & 5 & 361447.72 & 1.015 \\
\hline $6 s^{2} 6 p\left({ }^{2} P\right) 5 g$ & ${ }^{3} F^{o}$ & 2 & 361575.70 & 0.697 \\
\hline $6 s^{2} 6 p\left({ }^{2} P\right) 5 g$ & ${ }^{3} F^{o}$ & 3 & 362081.08 & 1.036 \\
\hline $6 s^{2} 6 p\left({ }^{2} P\right) 6 g$ & ${ }^{3} H^{o}$ & 4 & 350683.82 & 0.868 \\
\hline $6 s^{2} 6 p\left({ }^{2} P\right) 6 g$ & ${ }^{3} F^{o}$ & 4 & 350805.98 & 1.147 \\
\hline $6 s^{2} 6 p\left({ }^{2} P\right) 6 g$ & ${ }^{3} H^{o}$ & 5 & 350924.26 & 1.069 \\
\hline $6 s^{2} 6 p\left({ }^{2} P\right) 6 g$ & ${ }^{3} G^{o}$ & 3 & 350993.91 & 0.911 \\
\hline $6 s^{2} 6 p\left({ }^{2} P\right) 6 g$ & ${ }^{1} G^{o}$ & 4 & 394898.76 & 0.953 \\
\hline $6 s^{2} 6 p\left({ }^{2} P\right) 6 g$ & ${ }^{3} G^{o}$ & 5 & 395017.53 & 1.136 \\
\hline $6 s^{2} 6 p\left({ }^{2} P\right) 6 g$ & ${ }^{3} G^{o *}$ & 4 & 395329.30 & 1.128 \\
\hline $6 s^{2} 6 p\left({ }^{2} P\right) 6 g$ & ${ }^{3} F^{o *}$ & 3 & 395482.01 & 0.903 \\
\hline $6 s^{2} 6 p\left({ }^{2} P\right) 6 g$ & ${ }^{3} H^{o}$ & 6 & 395552.37 & 1.167 \\
\hline $6 s^{2} 6 p\left({ }^{2} P\right) 6 g$ & ${ }^{1} H^{o}$ & 5 & 396038.65 & 1.019 \\
\hline
\end{tabular}

\begin{tabular}{|c|c|c|c|c|}
\hline \multicolumn{3}{|c|}{ Levels } & $E\left[\mathrm{~cm}^{-1}\right]$ & $g$-factor \\
\hline Conf. & Term & $J$ & HFR & HFR \\
\hline $6 s^{2} 6 p\left({ }^{2} P\right) 6 g$ & ${ }^{3} F^{o}$ & 2 & 396050.80 & 0.666 \\
\hline $6 s^{2} 6 p\left({ }^{2} P\right) 6 g$ & ${ }^{1} F^{o}$ & 3 & 396347.73 & 1.024 \\
\hline $6 s^{2} 6 p\left({ }^{2} P\right) 7 g$ & ${ }^{3} H^{o}$ & 4 & 371620.50 & 0.861 \\
\hline $6 s^{2} 6 p\left({ }^{2} P\right) 7 g$ & ${ }^{3} F^{o}$ & 4 & 371732.69 & 1.154 \\
\hline $6 s^{2} 6 p\left({ }^{2} P\right) 7 g$ & ${ }^{3} H^{o}$ & 5 & 371812.78 & 1.068 \\
\hline $6 s^{2} 6 p\left({ }^{2} P\right) 7 g$ & ${ }^{3} F^{o *}$ & 3 & 371885.10 & 0.914 \\
\hline $6 s^{2} 6 p\left({ }^{2} P\right) 7 g$ & ${ }^{1} G^{o}$ & 4 & 416005.59 & 0.960 \\
\hline $6 s^{2} 6 p\left({ }^{2} P\right) 7 g$ & ${ }^{3} G^{o}$ & 5 & 416073.50 & 1.148 \\
\hline $6 s^{2} 6 p\left({ }^{2} P\right) 7 g$ & ${ }^{3} G^{o *}$ & 4 & 416242.21 & 1.124 \\
\hline $6 s^{2} 6 p\left({ }^{2} P\right) 7 g$ & ${ }^{3} G^{o}$ & 3 & 416326.48 & 0.893 \\
\hline $6 s^{2} 6 p\left({ }^{2} P\right) 7 g$ & ${ }^{3} H^{o}$ & 6 & 416402.19 & 1.167 \\
\hline $6 s^{2} 6 p\left({ }^{2} P\right) 7 g$ & ${ }^{3} F^{o}$ & 2 & 416718.70 & 0.666 \\
\hline $6 s^{2} 6 p\left({ }^{2} P\right) 7 g$ & ${ }^{1} H^{o}$ & 5 & 416724.09 & 1.018 \\
\hline $6 s^{2} 6 p\left({ }^{2} P\right) 7 g$ & ${ }^{1} F^{o}$ & 3 & 416922.80 & 1.026 \\
\hline $6 s^{2} 6 p\left({ }^{2} P\right) 8 g$ & ${ }^{3} H^{o}$ & 4 & 385144.49 & 0.863 \\
\hline $6 s^{2} 6 p\left({ }^{2} P\right) 8 g$ & ${ }^{3} F^{o}$ & 4 & 385212.96 & 1.153 \\
\hline $6 s^{2} 6 p\left({ }^{2} P\right) 8 g$ & ${ }^{3} H^{o}$ & 5 & 385266.62 & 1.068 \\
\hline $6 s^{2} 6 p\left({ }^{2} P\right) 8 g$ & ${ }^{3} F^{o *}$ & 3 & 385321.81 & 0.915 \\
\hline $6 s^{2} 6 p\left({ }^{2} P\right) 8 g$ & ${ }^{1} G^{o}$ & 4 & 429662.10 & 0.961 \\
\hline $6 s^{2} 6 p\left({ }^{2} P\right) 8 g$ & ${ }^{3} G^{o}$ & 5 & 429706.80 & 1.148 \\
\hline $6 s^{2} 6 p\left({ }^{2} P\right) 8 g$ & ${ }^{3} G^{o *}$ & 4 & 429813.41 & 1.124 \\
\hline $6 s^{2} 6 p\left({ }^{2} P\right) 8 g$ & ${ }^{3} G^{o}$ & 3 & 429868.27 & 0.893 \\
\hline $6 s^{2} 6 p\left({ }^{2} P\right) 8 g$ & ${ }^{3} H^{o}$ & 6 & 429912.49 & 1.167 \\
\hline $6 s^{2} 6 p\left({ }^{2} P\right) 8 g$ & ${ }^{3} F^{o}$ & 2 & 430125.11 & 0.666 \\
\hline $6 s^{2} 6 p\left({ }^{2} P\right) 8 g$ & ${ }^{1} H^{o}$ & 5 & 430138.58 & 1.018 \\
\hline $6 s^{2} 6 p\left({ }^{2} P\right) 8 g$ & ${ }^{1} F^{o}$ & 3 & 430275.39 & 1.025 \\
\hline $6 s^{2} 6 p\left({ }^{2} P\right) 9 g$ & ${ }^{3} H^{o}$ & 4 & 394344.20 & 0.962 \\
\hline $6 s^{2} 6 p\left({ }^{2} P\right) 9 g$ & ${ }^{3} G^{o}$ & 4 & 394375.26 & 1.059 \\
\hline $6 s^{2} 6 p\left({ }^{2} P\right) 9 g$ & ${ }^{3} F^{o *}$ & 3 & 394395.02 & 0.910 \\
\hline $6 s^{2} 6 p\left({ }^{2} P\right) 9 g$ & ${ }^{3} H^{o}$ & 5 & 394398.69 & 1.075 \\
\hline $6 s^{2} 6 p\left({ }^{2} P\right) 9 g$ & ${ }^{1} G^{o}$ & 4 & 438983.29 & 0.961 \\
\hline $6 s^{2} 6 p\left({ }^{2} P\right) 9 g$ & ${ }^{3} G^{o}$ & 5 & 439013.71 & 1.148 \\
\hline $6 s^{2} 6 p\left({ }^{2} P\right) 9 g$ & ${ }^{3} F^{o}$ & 4 & 439084.11 & 1.123 \\
\hline $6 s^{2} 6 p\left({ }^{2} P\right) 9 g$ & ${ }^{3} G^{o}$ & 3 & 439122.96 & 0.893 \\
\hline $6 s^{2} 6 p\left({ }^{2} P\right) 9 g$ & ${ }^{3} H^{o}$ & 6 & 439150.79 & 1.167 \\
\hline $6 s^{2} 6 p\left({ }^{2} P\right) 9 g$ & ${ }^{3} F^{o}$ & 2 & 439300.51 & 0.666 \\
\hline $6 s^{2} 6 p\left({ }^{2} P\right) 9 g$ & ${ }^{1} H^{o}$ & 5 & 439315.38 & 1.019 \\
\hline $6 s^{2} 6 p\left({ }^{2} P\right) 9 g$ & ${ }^{1} F^{o}$ & 3 & 439410.70 & 1.024 \\
\hline $6 s^{2} 6 p\left({ }^{2} P\right) 10 g$ & ${ }^{3} H^{o}$ & 4 & 401024.60 & 0.860 \\
\hline $6 s^{2} 6 p\left({ }^{2} P\right) 10 g$ & ${ }^{3} F^{o}$ & 4 & 401067.30 & 1.156 \\
\hline $6 s^{2} 6 p\left({ }^{2} P\right) 10 g$ & ${ }^{3} H^{o}$ & 5 & 401098.21 & 1.067 \\
\hline $6 s^{2} 6 p\left({ }^{2} P\right) 10 g$ & ${ }^{3} F^{o *}$ & 3 & 401128.26 & 0.915 \\
\hline $6 s^{2} 6 p\left({ }^{2} P\right) 10 g$ & ${ }^{1} G^{o}$ & 4 & 445661.10 & 0.961 \\
\hline $6 s^{2} 6 p\left({ }^{2} P\right) 10 g$ & ${ }^{3} G^{o}$ & 5 & 445682.21 & 1.148 \\
\hline $6 s^{2} 6 p\left({ }^{2} P\right) 10 g$ & ${ }^{3} G^{o}$ & 4 & 445730.86 & 1.123 \\
\hline $6 s^{2} 6 p\left({ }^{2} P\right) 10 g$ & ${ }^{3} G^{o}$ & 3 & 445758.81 & 0.893 \\
\hline $6 s^{2} 6 p\left({ }^{2} P\right) 10 g$ & ${ }^{3} H^{o}$ & 6 & 445777.38 & 1.167 \\
\hline $6 s^{2} 6 p\left({ }^{2} P\right) 10 g$ & ${ }^{3} F^{o}$ & 2 & 445888.20 & 0.666 \\
\hline $6 s^{2} 6 p\left({ }^{2} P\right) 10 g$ & ${ }^{1} H^{o}$ & 5 & 445901.90 & 1.019 \\
\hline
\end{tabular}


TABLE SI (cont.)

\begin{tabular}{|c|c|c|c|c|}
\hline \multicolumn{3}{|c|}{ Levels } & \multirow{2}{*}{$\frac{E\left[\mathrm{~cm}^{-1}\right]}{\mathrm{HFR}}$} & \multirow{2}{*}{$\frac{g \text {-factor }}{\text { HFR }}$} \\
\hline Conf. & Term & $J$ & & \\
\hline $6 s^{2} 6 p\left({ }^{2} P\right) 10 g$ & ${ }^{1} F^{o}$ & 3 & 445974.30 & 1.024 \\
\hline $6 s^{2} 6 p\left({ }^{2} P\right) 7 i$ & ${ }^{3} K^{o}$ & 6 & 372758.11 & 0.901 \\
\hline $6 s^{2} 6 p\left({ }^{2} P\right) 7 i$ & ${ }^{3} K^{o}$ & 7 & 372761.10 & 1.048 \\
\hline $6 s^{2} 6 p\left({ }^{2} P\right) 7 i$ & ${ }^{1} H^{o *}$ & 5 & 372768.40 & 0.944 \\
\hline $6 s^{2} 6 p\left({ }^{2} P\right) 7 i$ & ${ }^{3} H^{o}$ & 6 & 372769.37 & 1.107 \\
\hline $6 s^{2} 6 p\left({ }^{2} P\right) 7 i$ & ${ }^{1} I^{o}$ & 6 & 417385.70 & 0.967 \\
\hline $6 s^{2} 6 p\left({ }^{2} P\right) 7 i$ & ${ }^{3} I^{o}$ & 7 & 417388.41 & 1.105 \\
\hline $6 s^{2} 6 p\left({ }^{2} P\right) 7 i$ & ${ }^{3} I^{o}$ & 5 & 417436.98 & 0.905 \\
\hline $6 s^{2} 6 p\left({ }^{2} P\right) 7 i$ & ${ }^{3} I^{o *}$ & 6 & 417439.33 & 1.073 \\
\hline $6 s^{2} 6 p\left({ }^{2} P\right) 7 i$ & ${ }^{1} K^{o}$ & 7 & 417591.08 & 1.008 \\
\hline $6 s^{2} 6 p\left({ }^{2} P\right) 7 i$ & ${ }^{3} K^{o}$ & 8 & 417593.08 & 1.125 \\
\hline $6 s^{2} 6 p\left({ }^{2} P\right) 7 i$ & ${ }^{3} H^{o}$ & 4 & 417641.50 & 0.800 \\
\hline $6 s^{2} 6 p\left({ }^{2} P\right) 7 i$ & ${ }^{3} H^{o}$ & 5 & 417644.48 & 1.018 \\
\hline $6 s^{2} 6 p\left({ }^{2} P\right) 8 i$ & ${ }^{3} K^{o}$ & 6 & 385916.11 & 0.901 \\
\hline $6 s^{2} 6 p\left({ }^{2} P\right) 8 i$ & ${ }^{3} K^{o}$ & 7 & 385917.94 & 0.944 \\
\hline $6 s^{2} 6 p\left({ }^{2} P\right) 8 i$ & ${ }^{1} H^{o *}$ & 5 & 385921.20 & 1.107 \\
\hline $6 s^{2} 6 p\left({ }^{2} P\right) 8 i$ & ${ }^{3} H^{o}$ & 6 & 385921.64 & 1.048 \\
\hline $6 s^{2} 6 p\left({ }^{2} P\right) 8 i$ & ${ }^{1} I^{o}$ & 6 & 430583.05 & 0.967 \\
\hline $6 s^{2} 6 p\left({ }^{2} P\right) 8 i$ & ${ }^{3} I^{o}$ & 7 & 430584.56 & 1.105 \\
\hline $6 s^{2} 6 p\left({ }^{2} P\right) 8 i$ & ${ }^{3} I^{o}$ & 5 & 430617.68 & 0.904 \\
\hline $6 s^{2} 6 p\left({ }^{2} P\right) 8 i$ & ${ }^{3} I^{o *}$ & 6 & 430618.70 & 1.073 \\
\hline $6 s^{2} 6 p\left({ }^{2} P\right) 8 i$ & ${ }^{1} K^{o}$ & 7 & 430721.41 & 1.008 \\
\hline $6 s^{2} 6 p\left({ }^{2} P\right) 8 i$ & ${ }^{3} K^{o}$ & 8 & 430721.86 & 1.125 \\
\hline $6 s^{2} 6 p\left({ }^{2} P\right) 8 i$ & ${ }^{3} H^{o}$ & 4 & 430755.53 & 0.800 \\
\hline $6 s^{2} 6 p\left({ }^{2} P\right) 8 i$ & ${ }^{3} H^{o}$ & 5 & 430757.40 & 1.018 \\
\hline $6 s^{2} 6 p\left({ }^{2} P\right) 9 i$ & ${ }^{1} H^{o *}$ & 5 & 394950.99 & 0.947 \\
\hline $6 s^{2} 6 p\left({ }^{2} P\right) 9 i$ & ${ }^{3} K^{o}$ & 6 & 394951.95 & 0.927 \\
\hline $6 s^{2} 6 p\left({ }^{2} P\right) 9 i$ & ${ }^{3} H^{o}$ & 6 & 394952.30 & 1.082 \\
\hline $6 s^{2} 6 p\left({ }^{2} P\right) 9 i$ & ${ }^{3} K^{o}$ & 7 & 394953.85 & 1.048 \\
\hline $6 s^{2} 6 p\left({ }^{2} P\right) 9 i$ & ${ }^{1} I^{o}$ & 6 & 439643.59 & 0.967 \\
\hline $6 s^{2} 6 p\left({ }^{2} P\right) 9 i$ & ${ }^{3} I^{o}$ & 7 & 439645.10 & 1.105 \\
\hline $6 s^{2} 6 p\left({ }^{2} P\right) 9 i$ & ${ }^{3} I^{o}$ & 5 & 439667.90 & 0.904 \\
\hline $6 s^{2} 6 p\left({ }^{2} P\right) 9 i$ & ${ }^{3} I^{o *}$ & 6 & 439668.92 & 1.073 \\
\hline $6 s^{2} 6 p\left({ }^{2} P\right) 9 i$ & ${ }^{1} K^{o}$ & 7 & 439740.93 & 1.008 \\
\hline $6 s^{2} 6 p\left({ }^{2} P\right) 9 i$ & ${ }^{3} K^{o}$ & 8 & 439741.34 & 1.125 \\
\hline $6 s^{2} 6 p\left({ }^{2} P\right) 9 i$ & ${ }^{3} H^{o}$ & 4 & 439765.08 & 0.800 \\
\hline $6 s^{2} 6 p\left({ }^{2} P\right) 9 i$ & ${ }^{3} H^{o}$ & 5 & 439766.97 & 1.018 \\
\hline $6 s^{2} 6 p\left({ }^{2} P\right) 10 i$ & ${ }^{3} K^{o}$ & 6 & 401414.45 & 0.901 \\
\hline $6 s^{2} 6 p\left({ }^{2} P\right) 10 i$ & ${ }^{3} K^{o}$ & 7 & 401415.60 & 1.048 \\
\hline $6 s^{2} 6 p\left({ }^{2} P\right) 10 i$ & ${ }^{3} H^{o}$ & 6 & 401417.56 & 1.107 \\
\hline $6 s^{2} 6 p\left({ }^{2} P\right) 10 i$ & ${ }^{1} H^{o *}$ & 5 & 401417.99 & 0.944 \\
\hline $6 s^{2} 6 p\left({ }^{2} P\right) 10 i$ & ${ }^{1} I^{o}$ & 6 & 446122.40 & 0.967 \\
\hline $6 s^{2} 6 p\left({ }^{2} P\right) 10 i$ & ${ }^{3} I^{o}$ & 7 & 446123.25 & 1.105 \\
\hline $6 s^{2} 6 p\left({ }^{2} P\right) 10 i$ & ${ }^{3} I^{o}$ & 5 & 446139.97 & 0.904 \\
\hline $6 s^{2} 6 p\left({ }^{2} P\right) 10 i$ & ${ }^{3} I^{o *}$ & 6 & 446140.41 & 1.073 \\
\hline $6 s^{2} 6 p\left({ }^{2} P\right) 10 i$ & ${ }^{3} K^{o}$ & 8 & 446193.13 & 1.125 \\
\hline $6 s^{2} 6 p\left({ }^{2} P\right) 10 i$ & ${ }^{1} K^{o}$ & 7 & 446193.28 & 1.008 \\
\hline $6 s^{2} 6 p\left({ }^{2} P\right) 10 i$ & ${ }^{3} H^{o}$ & 4 & 446211.13 & 0.800 \\
\hline $6 s^{2} 6 p\left({ }^{2} P\right) 10 i$ & ${ }^{3} H^{o}$ & 5 & 446212.36 & 1.018 \\
\hline
\end{tabular}


TABLE SII

The wavelengths, $\lambda$, logarithmic weighted oscillator strengths, $\log (g f)$, and transition probabilities, $A_{j i}$, for E1 transitions in Rn V. Numbers in brackets represent powers of 10 .

\begin{tabular}{|c|c|c|c|c|c|c|c|c|c|}
\hline \multicolumn{4}{|c|}{ Transitions } & \multicolumn{2}{|c|}{$\lambda[\AA]$} & \multicolumn{2}{|c|}{$\log (g f)$} & \multicolumn{2}{|c|}{$A_{j i}\left[\mathrm{~s}^{-1}\right]$} \\
\hline Lower level & Term & Upper level & Term & HFR & MCDF & HFR & MCDF & HFR & MCDF \\
\hline $6 s^{2} 6 p^{2}$ & ${ }^{3} P_{0}$ & $6 s^{2} 6 p\left({ }^{2} P\right) 6 d$ & ${ }^{3} D_{1}^{o}$ & 555.51 & 554.41 & 0.402 & 0.374 & $1.82(10)$ & $1.71(10)$ \\
\hline $6 s^{2} 6 p^{2}$ & ${ }^{3} P_{1}$ & $6 s^{2} 6 p\left({ }^{2} P\right) 6 d$ & ${ }^{3} P_{1}^{o}$ & 541.22 & 536.58 & 0.293 & 0.214 & $1.49(10)$ & $1.26(10)$ \\
\hline $6 s^{2} 6 p^{2}$ & ${ }^{3} P_{1}$ & $6 s^{2} 6 p\left({ }^{2} P\right) 6 d$ & ${ }^{3} P_{2}^{o}$ & 693.57 & 687.87 & 0.491 & 0.521 & $8.59(9)$ & $9.36(9)$ \\
\hline $6 s^{2} 6 p^{2}$ & ${ }^{3} P_{1}$ & $6 s^{2} 6 p\left({ }^{2} P\right) 6 d$ & ${ }^{1} D_{2}^{o}$ & 558.86 & 561.02 & 0.271 & 0.236 & $7.96(9)$ & $7.30(9)$ \\
\hline $6 s^{2} 6 p^{2}$ & ${ }^{3} P_{1}$ & $6 s^{2} 6 p\left({ }^{2} P\right) 6 d$ & ${ }^{3} P_{0}^{o}$ & 541.78 & 538.10 & -0.106 & -0.176 & $1.78(10)$ & $1.54(10)$ \\
\hline $6 s^{2} 6 p^{2}$ & ${ }^{3} P_{1}$ & $6 s^{2} 6 p\left({ }^{2} P\right) 7 s$ & ${ }^{3} P_{2}^{o}$ & 511.31 & 515.81 & -0.176 & -0.446 & $3.40(9)$ & $1.80(9)$ \\
\hline $6 s^{2} 6 p^{2}$ & ${ }^{3} P_{1}$ & $6 s^{2} 6 p\left({ }^{2} P\right) 6 d$ & ${ }^{3} P_{2}^{o}$ & 536.68 & 531.96 & -0.320 & -0.332 & $2.22(9)$ & $2.19(9)$ \\
\hline $6 s^{2} 6 p^{2}$ & ${ }^{3} P_{1}$ & $6 s^{2} 6 p\left({ }^{2} P\right) 7 s$ & ${ }^{3} P_{0}^{o}$ & 657.15 & 663.03 & -0.367 & -0.269 & $6.64(9)$ & $8.16(9)$ \\
\hline $6 s^{2} 6 p^{2}$ & ${ }^{3} P_{1}$ & $6 s^{2} 6 p\left({ }^{2} P\right) 6 d$ & ${ }^{3} D_{1}^{o}$ & 688.62 & 678.64 & -0.348 & -0.321 & $2.10(9)$ & $2.30(9)$ \\
\hline $6 s^{2} 6 p^{2}$ & ${ }^{3} P_{1}$ & $6 s^{2} 6 p\left({ }^{2} P\right) 7 s$ & ${ }^{3} P_{1}^{o}$ & 651.85 & 658.87 & -1.008 & -0.767 & $5.14(8)$ & $8.75(8)$ \\
\hline $6 s^{2} 6 p^{2}$ & ${ }^{3} P_{1}$ & $6 s^{2} 6 p\left({ }^{2} P\right) 6 d$ & ${ }^{3} F_{2}^{o}$ & 751.72 & 753.78 & -1.384 & -1.380 & $9.75(7)$ & $9.80(7)$ \\
\hline $6 s^{2} 6 p^{2}$ & ${ }^{1} D_{2}$ & $6 s^{2} 6 p\left({ }^{2} P\right) 6 d$ & ${ }^{3} D_{3}^{o}$ & 562.39 & 564.69 & 0.760 & 0.729 & $1.73(10)$ & $1.60(10)$ \\
\hline $6 s^{2} 6 p^{2}$ & ${ }^{1} D_{2}$ & $6 s^{2} 6 p\left({ }^{2} P\right) 6 d$ & ${ }^{1} F_{3}^{o}$ & 522.98 & 512.36 & 0.282 & 0.208 & $6.66(9)$ & $5.86(9)$ \\
\hline $6 s^{2} 6 p^{2}$ & ${ }^{1} D_{2}$ & $6 s^{2} 6 p\left({ }^{2} P\right) 6 d$ & ${ }^{3} F_{3}^{o}$ & 719.87 & 725.90 & 0.477 & 0.478 & $5.52(9)$ & $5.43(9)$ \\
\hline $6 s^{2} 6 p^{2}$ & ${ }^{1} D_{2}$ & $6 s^{2} 6 p\left({ }^{2} P\right) 6 d$ & ${ }^{1} D_{2}^{o}$ & 575.48 & 583.07 & 0.082 & -0.107 & $4.87(9)$ & $3.07(9)$ \\
\hline $6 s^{2} 6 p^{2}$ & ${ }^{1} D_{2}$ & $6 s^{2} 6 p\left({ }^{2} P\right) 6 d$ & ${ }^{3} P_{2}^{o}$ & 551.99 & 551.75 & 0.023 & 0.120 & $4.61(9)$ & $5.71(9)$ \\
\hline $6 s^{2} 6 p^{2}$ & ${ }^{1} D_{2}$ & $6 s^{2} 6 p\left({ }^{2} P\right) 7 s$ & ${ }^{3} P_{2}^{o}$ & 525.18 & 534.40 & -0.133 & -0.263 & $3.56(9)$ & $2.55(9)$ \\
\hline $6 s^{2} 6 p^{2}$ & ${ }^{1} D_{2}$ & $6 s^{2} 6 p\left({ }^{2} P\right) 7 s$ & ${ }^{3} P_{1}^{o}$ & 674.57 & 689.49 & -0.014 & 0.095 & $4.73(9)$ & $5.82(9)$ \\
\hline $6 s^{2} 6 p^{2}$ & ${ }^{1} D_{2}$ & $6 s^{2} 6 p\left({ }^{2} P\right) 7 s$ & ${ }^{1} P_{1}^{o}$ & 504.48 & 538.47 & -0.385 & -0.883 & $3.60(9)$ & $1.00(9)$ \\
\hline $6 s^{2} 6 p^{2}$ & ${ }^{1} D_{2}$ & $6 s^{2} 6 p\left({ }^{2} P\right) 6 d$ & ${ }^{3} P_{1}^{o}$ & 556.79 & 556.72 & -0.400 & -0.439 & $2.86(9)$ & $2.61(9)$ \\
\hline $6 s^{2} 6 p^{2}$ & ${ }^{1} D_{2}$ & $6 s^{2} 6 p\left({ }^{2} P\right) 6 d$ & ${ }^{3} P_{2}^{o}$ & 719.36 & 721.31 & -0.305 & -0.241 & $1.28(9)$ & $1.47(9)$ \\
\hline $6 s^{2} 6 p^{2}$ & ${ }^{1} D_{2}$ & $6 s^{2} 6 p\left({ }^{2} P\right) 6 d$ & ${ }^{1} P_{1}^{o}$ & 532.28 & 495.99 & -0.658 & -0.653 & $1.72(9)$ & $2.01(9)$ \\
\hline $6 s^{2} 6 p^{2}$ & ${ }^{1} D_{2}$ & $6 s^{2} 6 p\left({ }^{2} P\right) 6 d$ & ${ }^{3} F_{2}^{o}$ & 782.10 & 794.14 & -0.336 & -0.304 & $1.01(9)$ & $1.05(9)$ \\
\hline $6 s^{2} 6 p^{2}$ & ${ }^{3} P_{2}$ & $6 s^{2} 6 p\left({ }^{2} P\right) 6 d$ & ${ }^{1} F_{3}^{o}$ & 657.70 & 634.59 & 0.924 & 0.958 & $1.85(10)$ & $2.15(10)$ \\
\hline $6 s^{2} 6 p^{2}$ & ${ }^{3} P_{2}$ & $6 s^{2} 6 p\left({ }^{2} P\right) 6 d$ & ${ }^{3} P_{2}^{o}$ & 704.25 & 696.14 & 0.467 & 0.459 & $7.88(9)$ & 7.93(9) \\
\hline $6 s^{2} 6 p^{2}$ & ${ }^{3} P_{2}$ & $6 s^{2} 6 p\left({ }^{2} P\right) 7 s$ & ${ }^{1} P_{1}^{o}$ & 628.70 & 675.14 & -0.048 & -0.242 & $5.03(9)$ & $2.79(9)$ \\
\hline $6 s^{2} 6 p^{2}$ & ${ }^{3} P_{2}$ & $6 s^{2} 6 p\left({ }^{2} P\right) 6 d$ & ${ }^{3} D_{3}^{o}$ & 721.26 & 716.88 & -0.043 & 0.043 & $1.66(9)$ & $2.05(9)$ \\
\hline $6 s^{2} 6 p^{2}$ & ${ }^{3} P_{2}$ & $6 s^{2} 6 p\left({ }^{2} P\right) 7 s$ & ${ }^{3} P_{2}^{o}$ & 661.19 & 668.74 & -0.146 & -0.039 & $2.18(9)$ & $2.73(9)$ \\
\hline $6 s^{2} 6 p^{2}$ & ${ }^{3} P_{2}$ & $6 s^{2} 6 p\left({ }^{2} P\right) 6 d$ & ${ }^{3} P_{1}^{o}$ & 712.08 & 704.08 & -0.271 & -0.208 & $2.35(9)$ & $2.78(9)$ \\
\hline $6 s^{2} 6 p^{2}$ & ${ }^{3} P_{2}$ & $6 s^{2} 6 p\left({ }^{2} P\right) 6 d$ & ${ }^{1} P_{1}^{o}$ & 672.48 & 609.67 & -0.563 & -0.039 & $1.35(9)$ & $5.47(9)$ \\
\hline $6 s^{2} 6 p^{2}$ & ${ }^{3} P_{2}$ & $6 s^{2} 6 p\left({ }^{2} P\right) 6 d$ & ${ }^{1} D_{2}^{o}$ & 742.93 & 746.75 & -0.784 & -0.599 & $3.98(8)$ & $6.02(8)$ \\
\hline $6 s^{2} 6 p^{2}$ & ${ }^{3} P_{2}$ & $6 s^{2} 6 p\left({ }^{2} P\right) 6 d$ & ${ }^{3} P_{2}^{o}$ & 1001.55 & 989.68 & -1.788 & -1.564 & $2.17(7)$ & $3.71(7)$ \\
\hline $6 s^{2} 6 p^{2}$ & ${ }^{3} P_{2}$ & $6 s^{2} 6 p\left({ }^{2} P\right) 6 d$ & ${ }^{3} D_{1}^{o}$ & 991.23 & 970.68 & -1.870 & -1.634 & $3.05(7)$ & $5.48(7)$ \\
\hline $6 s^{2} 6 p^{2}$ & ${ }^{3} P_{2}$ & $6 s^{2} 6 p\left({ }^{2} P\right) 6 d$ & ${ }^{3} F_{2}^{o}$ & 1127.48 & 1132.10 & -1.839 & -1.566 & $1.52(7)$ & $2.83(7)$ \\
\hline $6 s^{2} 6 p\left({ }^{2} P\right) 6 d$ & ${ }^{3} F_{2}^{o}$ & $6 s^{2} 6 p\left({ }^{2} P\right) 7 p$ & ${ }^{3} D_{1}$ & 1857.23 & 1945.60 & 0.064 & 0.134 & $7.47(8)$ & $8.00(8)$ \\
\hline $6 s^{2} 6 p\left({ }^{2} P\right) 7 s$ & ${ }^{3} P_{1}^{o}$ & $6 s^{2} 6 p\left({ }^{2} P\right) 7 p$ & ${ }^{3} D_{1}$ & 2988.37 & 3097.10 & -0.169 & -0.111 & $1.69(8)$ & $1.79(8)$ \\
\hline $6 s^{2} 6 p\left({ }^{2} P\right) 6 d$ & ${ }^{3} D_{1}^{o}$ & $6 s^{2} 6 p\left({ }^{2} P\right) 7 p$ & ${ }^{3} D_{1}$ & 2400.81 & 2724.10 & -0.363 & -0.420 & $1.67(8)$ & $1.14(8)$ \\
\hline $6 s^{2} 6 p\left({ }^{2} P\right) 7 s$ & ${ }^{3} P_{0}^{o}$ & $6 s^{2} 6 p\left({ }^{2} P\right) 7 p$ & ${ }^{3} D_{1}$ & 2881.83 & 3008.40 & -0.356 & -0.348 & $1.18(8)$ & $1.10(8)$ \\
\hline $6 s^{2} 6 p\left({ }^{2} P\right) 7 s$ & ${ }^{3} P_{1}^{o}$ & $6 s^{2} 6 p\left({ }^{2} P\right) 7 p$ & ${ }^{3} P_{0}$ & 2570.38 & 2577.10 & -0.175 & -0.205 & $6.74(8)$ & $6.27(8)$ \\
\hline $6 s^{2} 6 p\left({ }^{2} P\right) 6 d$ & ${ }^{3} D_{1}^{o}$ & $6 s^{2} 6 p\left({ }^{2} P\right) 7 p$ & ${ }^{3} P_{0}$ & 2123.39 & 2313.50 & -0.573 & -0.448 & $3.95(8)$ & $4.44(8)$ \\
\hline $6 s^{2} 6 p\left({ }^{2} P\right) 6 d$ & ${ }^{3} P_{2}^{o}$ & $6 s^{2} 6 p\left({ }^{2} P\right) 7 p$ & ${ }^{3} P_{1}$ & 1772.61 & 1910.00 & 0.155 & 0.138 & 10.1(8) & $8.41(8)$ \\
\hline $6 s^{2} 6 p\left({ }^{2} P\right) 7 s$ & ${ }^{3} P_{0}^{o}$ & $6 s^{2} 6 p\left({ }^{2} P\right) 7 p$ & ${ }^{3} P_{1}$ & 2065.17 & 2130.00 & 0.093 & 0.075 & $6.45(8)$ & $5.85(8)$ \\
\hline $6 s^{2} 6 p\left({ }^{2} P\right) 7 s$ & ${ }^{3} P_{1}^{o}$ & $6 s^{2} 6 p\left({ }^{2} P\right) 7 p$ & ${ }^{3} P_{1}$ & 2119.31 & 2170.00 & -0.326 & -0.300 & $2.34(8)$ & $2.37(8)$ \\
\hline $6 s^{2} 6 p\left({ }^{2} P\right) 6 d$ & ${ }^{3} D_{1}^{o}$ & $6 s^{2} 6 p\left({ }^{2} P\right) 7 p$ & ${ }^{3} P_{1}$ & 1805.88 & 1980.00 & -0.547 & -0.700 & $1.93(8)$ & $1.13(8)$ \\
\hline $6 s^{2} 6 p\left({ }^{2} P\right) 6 d$ & ${ }^{3} F_{2}^{o}$ & $6 s^{2} 6 p\left({ }^{2} P\right) 7 p$ & ${ }^{3} P_{1}$ & 1480.03 & 1540.00 & -1.510 & -1.545 & $3.17(7)$ & $2.69(7)$ \\
\hline $6 s^{2} 6 p\left({ }^{2} P\right) 6 d$ & ${ }^{3} F_{3}^{o}$ & $6 s^{2} 6 p\left({ }^{2} P\right) 7 p$ & ${ }^{3} D_{2}$ & 1759.84 & 1860.00 & 0.329 & 0.312 & $9.18(8)$ & $7.94(8)$ \\
\hline $6 s^{2} 6 p\left({ }^{2} P\right) 7 s$ & ${ }^{3} P_{1}^{o}$ & $6 s^{2} 6 p\left({ }^{2} P\right) 7 p$ & ${ }^{3} D_{2}$ & 2105.47 & 2140.00 & 0.470 & 0.456 & $8.88(8)$ & $8.29(8)$ \\
\hline $6 s^{2} 6 p\left({ }^{2} P\right) 6 d$ & ${ }^{3} F_{2}^{o}$ & $6 s^{2} 6 p\left({ }^{2} P\right) 7 p$ & ${ }^{3} D_{2}$ & 1473.27 & 1520.00 & -0.581 & -0.614 & $1.61(8)$ & $1.40(8)$ \\
\hline
\end{tabular}


TABLE SII (cont.)

\begin{tabular}{|c|c|c|c|c|c|c|c|c|c|}
\hline \multicolumn{4}{|c|}{ Transitions } & \multicolumn{2}{|c|}{$\lambda[\AA]$} & \multicolumn{2}{|c|}{$\log (g f)$} & \multicolumn{2}{|c|}{$A_{j i}\left[\mathrm{~s}^{-1}\right]$} \\
\hline Lower level & Term & Upper level & Term & HFR & MCDF & HFR & MCDF & HFR & MCDF \\
\hline $6 s^{2} 6 p\left({ }^{2} P\right) 6 d$ & ${ }^{3} P_{2}^{o}$ & $6 s^{2} 6 p\left({ }^{2} P\right) 7 p$ & ${ }^{3} D_{2}$ & 1762.92 & 1890.00 & -0.814 & -0.817 & $6.59(7)$ & $5.72(7)$ \\
\hline $6 s^{2} 6 p\left({ }^{2} P\right) 6 d$ & ${ }^{3} D_{3}^{o}$ & $6 s^{2} 6 p\left({ }^{2} P\right) 7 p$ & ${ }^{1} D_{2}$ & 1971.70 & 2112.00 & 0.084 & 0.100 & $4.17(8)$ & $3.76(8)$ \\
\hline $6 s^{2} 6 p\left({ }^{2} P\right) 6 d$ & ${ }^{1} D_{2}^{o}$ & $6 s^{2} 6 p\left({ }^{2} P\right) 7 p$ & ${ }^{1} D_{2}$ & 1825.20 & 1889.30 & -0.367 & -0.430 & $1.72(8)$ & $1.39(8)$ \\
\hline $6 s^{2} 6 p\left({ }^{2} P\right) 6 d$ & ${ }^{3} P_{2}^{o}$ & $6 s^{2} 6 p\left({ }^{2} P\right) 7 p$ & ${ }^{1} D_{2}$ & 2109.97 & 2315.20 & -0.517 & -0.399 & $9.10(7)$ & $9.92(7)$ \\
\hline $6 s^{2} 6 p\left({ }^{2} P\right) 6 d$ & ${ }^{1} F_{3}^{o}$ & $6 s^{2} 6 p\left({ }^{2} P\right) 7 p$ & ${ }^{1} D_{2}$ & 2677.04 & 3417.60 & -0.520 & -0.679 & $5.62(7)$ & $3.35(7)$ \\
\hline $6 s^{2} 6 p\left({ }^{2} P\right) 6 d$ & ${ }^{3} D_{1}^{o}$ & $6 s^{2} 6 p\left({ }^{2} P\right) 7 p$ & ${ }^{1} D_{2}$ & 1129.88 & 1193.00 & -1.285 & -1.854 & $5.42(7)$ & $1.31(7)$ \\
\hline $6 s^{2} 6 p\left({ }^{2} P\right) 6 d$ & ${ }^{3} F_{3}^{o}$ & $6 s^{2} 6 p\left({ }^{2} P\right) 7 p$ & ${ }^{1} D_{2}$ & 1115.53 & 1153.70 & -1.396 & -0.998 & $4.31(7)$ & $1.01(8)$ \\
\hline $6 s^{2} 6 p\left({ }^{2} P\right) 6 d$ & ${ }^{3} P_{1}^{o}$ & $6 s^{2} 6 p\left({ }^{2} P\right) 7 p$ & ${ }^{1} D_{2}$ & 2042.61 & 2231.60 & -1.115 & -1.047 & $2.45(7)$ & $2.40(7)$ \\
\hline $6 s^{2} 6 p\left({ }^{2} P\right) 6 d$ & ${ }^{3} P_{2}^{o}$ & $6 s^{2} 6 p\left({ }^{2} P\right) 7 p$ & ${ }^{1} D_{2}$ & 1116.76 & 1165.50 & -1.786 & -1.704 & $1.75(7)$ & $1.94(7)$ \\
\hline $6 s^{2} 6 p\left({ }^{2} P\right) 7 s$ & ${ }^{3} P_{1}^{o}$ & $6 s^{2} 6 p\left({ }^{2} P\right) 7 p$ & ${ }^{1} D_{2}$ & 1245.09 & 1259.40 & -2.365 & -2.207 & $3.71(6)$ & $5.22(6)$ \\
\hline $6 s^{2} 6 p\left({ }^{2} P\right) 6 d$ & ${ }^{3} F_{4}^{o}$ & $6 s^{2} 6 p\left({ }^{2} P\right) 7 p$ & ${ }^{3} D_{3}$ & 1560.54 & 1610.00 & 0.465 & 0.455 & 1.14(9) & $1.05(9)$ \\
\hline $6 s^{2} 6 p\left({ }^{2} P\right) 7 s$ & ${ }^{3} P_{2}^{o}$ & $6 s^{2} 6 p\left({ }^{2} P\right) 7 p$ & ${ }^{3} D_{3}$ & 2111.99 & 2190.00 & 0.623 & 0.601 & $8.98(8)$ & $7.93(8)$ \\
\hline $6 s^{2} 6 p\left({ }^{2} P\right) 6 d$ & ${ }^{3} D_{3}^{o}$ & $6 s^{2} 6 p\left({ }^{2} P\right) 7 p$ & ${ }^{3} D_{3}$ & 1668.20 & 1800.00 & -0.134 & -0.142 & $2.52(8)$ & $2.13(8)$ \\
\hline $6 s^{2} 6 p\left({ }^{2} P\right) 6 d$ & ${ }^{3} P_{2}^{o}$ & $6 s^{2} 6 p\left({ }^{2} P\right) 7 p$ & ${ }^{3} D_{3}$ & 1012.70 & 1060.00 & -1.317 & -0.831 & $4.47(7)$ & $1.24(8)$ \\
\hline $6 s^{2} 6 p\left({ }^{2} P\right) 6 d$ & ${ }^{1} D_{2}^{o}$ & $6 s^{2} 6 p\left({ }^{2} P\right) 7 p$ & ${ }^{3} D_{3}$ & 1562.74 & 1630.00 & -1.481 & -1.264 & $1.29(7)$ & $1.96(7)$ \\
\hline $6 s^{2} 6 p\left({ }^{2} P\right) 6 d$ & ${ }^{1} F_{3}^{o}$ & $6 s^{2} 6 p\left({ }^{2} P\right) 7 p$ & ${ }^{3} D_{3}$ & 2148.34 & 2660.00 & -1.264 & -1.328 & $11.2(6)$ & $6.33(6)$ \\
\hline $6 s^{2} 6 p\left({ }^{2} P\right) 6 d$ & ${ }^{3} P_{2}^{o}$ & $6 s^{2} 6 p\left({ }^{2} P\right) 7 p$ & ${ }^{3} D_{3}$ & 1766.92 & 1939.80 & -1.534 & -1.627 & $8.93(6)$ & $5.98(6)$ \\
\hline $6 s^{2} 6 p\left({ }^{2} P\right) 6 d$ & ${ }^{3} F_{3}^{o}$ & $6 s^{2} 6 p\left({ }^{2} P\right) 7 p$ & ${ }^{3} D_{3}$ & 1011.69 & 1050.00 & -2.630 & -2.642 & $2.18(6)$ & $1.96(6)$ \\
\hline $6 s^{2} 6 p\left({ }^{2} P\right) 6 d$ & ${ }^{1} D_{2}^{o}$ & $6 s^{2} 6 p\left({ }^{2} P\right) 7 p$ & ${ }^{3} S_{1}$ & 1545.73 & 1600.00 & -0.351 & -0.409 & $4.15(8)$ & $3.38(8)$ \\
\hline $6 s^{2} 6 p\left({ }^{2} P\right) 6 d$ & ${ }^{3} P_{2}^{o}$ & $6 s^{2} 6 p\left({ }^{2} P\right) 7 p$ & ${ }^{3} S_{1}$ & 1745.21 & 1896.20 & -0.291 & -0.294 & $3.74(8)$ & $3.14(8)$ \\
\hline $6 s^{2} 6 p\left({ }^{2} P\right) 7 s$ & ${ }^{3} P_{2}^{o}$ & $6 s^{2} 6 p\left({ }^{2} P\right) 7 p$ & ${ }^{3} S_{1}$ & 2081.04 & 2130.00 & -0.207 & -0.137 & $3.19(8)$ & $3.55(8)$ \\
\hline $6 s^{2} 6 p\left({ }^{2} P\right) 6 d$ & ${ }^{3} P_{0}^{o}$ & $6 s^{2} 6 p\left({ }^{2} P\right) 7 p$ & ${ }^{3} S_{1}$ & 1693.39 & 1820.00 & -0.959 & -0.917 & $8.52(7)$ & $8.09(7)$ \\
\hline $6 s^{2} 6 p\left({ }^{2} P\right) 6 d$ & ${ }^{3} P_{1}^{o}$ & $6 s^{2} 6 p\left({ }^{2} P\right) 7 p$ & ${ }^{3} S_{1}$ & 1698.87 & 1840.00 & -0.960 & -0.790 & $8.45(7)$ & $10.6(7)$ \\
\hline $6 s^{2} 6 p\left({ }^{2} P\right) 7 s$ & ${ }^{3} P_{1}^{o}$ & $6 s^{2} 6 p\left({ }^{2} P\right) 7 p$ & ${ }^{3} S_{1}$ & 1108.39 & 1120.00 & -2.667 & -2.437 & $3.90(6)$ & $6.44(6)$ \\
\hline $6 s^{2} 6 p\left({ }^{2} P\right) 6 d$ & ${ }^{3} P_{2}^{o}$ & $6 s^{2} 6 p\left({ }^{2} P\right) 7 p$ & ${ }^{3} S_{1}$ & 1005.53 & 1050.00 & -2.853 & -2.447 & $3.08(6)$ & $7.22(6)$ \\
\hline $6 s^{2} 6 p\left({ }^{2} P\right) 6 d$ & ${ }^{3} D_{1}^{o}$ & $6 s^{2} 6 p\left({ }^{2} P\right) 7 p$ & ${ }^{3} S_{1}$ & 1016.15 & 1070.00 & -3.201 & -2.732 & $1.35(6)$ & $3.59(6)$ \\
\hline $6 s^{2} 6 p\left({ }^{2} P\right) 7 s$ & ${ }^{3} P_{0}^{o}$ & $6 s^{2} 6 p\left({ }^{2} P\right) 7 p$ & ${ }^{3} S_{1}$ & 1093.39 & 1110.00 & -3.194 & -3.095 & $1.19(6)$ & $1.44(6)$ \\
\hline $6 s^{2} 6 p\left({ }^{2} P\right) 6 d$ & ${ }^{1} F_{3}^{o}$ & $6 s^{2} 6 p\left({ }^{2} P\right) 7 p$ & ${ }^{3} P_{2}$ & 2001.68 & 2392.40 & 0.240 & 0.204 & $5.79(8)$ & $3.73(8)$ \\
\hline $6 s^{2} 6 p\left({ }^{2} P\right) 7 s$ & ${ }^{3} P_{2}^{o}$ & $6 s^{2} 6 p\left({ }^{2} P\right) 7 p$ & ${ }^{3} P_{2}$ & 1970.09 & 2006.20 & 0.091 & 0.001 & $4.24(8)$ & $3.32(8)$ \\
\hline $6 s^{2} 6 p\left({ }^{2} P\right) 6 d$ & ${ }^{3} P_{2}^{o}$ & $6 s^{2} 6 p\left({ }^{2} P\right) 7 p$ & ${ }^{3} P_{2}$ & 1666.50 & 1794.30 & -0.147 & -0.284 & $3.42(8)$ & $2.15(8)$ \\
\hline $6 s^{2} 6 p\left({ }^{2} P\right) 7 s$ & ${ }^{1} P_{1}^{o}$ & $6 s^{2} 6 p\left({ }^{2} P\right) 7 p$ & ${ }^{3} P_{2}$ & 2328.59 & 1950.70 & -0.057 & 0.070 & $2.16(8)$ & $4.12(8)$ \\
\hline $6 s^{2} 6 p\left({ }^{2} P\right) 6 d$ & ${ }^{3} D_{1}^{o}$ & $6 s^{2} 6 p\left({ }^{2} P\right) 7 p$ & ${ }^{3} P_{2}$ & 988.95 & 1037.80 & -1.010 & -1.702 & $1.33(8)$ & $2.46(7)$ \\
\hline $6 s^{2} 6 p\left({ }^{2} P\right) 6 d$ & ${ }^{3} D_{3}^{o}$ & $6 s^{2} 6 p\left({ }^{2} P\right) 7 p$ & ${ }^{3} P_{2}$ & 1578.40 & 1669.80 & -0.718 & -0.806 & $1.02(8)$ & $7.49(7)$ \\
\hline $6 s^{2} 6 p\left({ }^{2} P\right) 6 d$ & ${ }^{3} P_{1}^{o}$ & $6 s^{2} 6 p\left({ }^{2} P\right) 7 p$ & ${ }^{3} P_{2}$ & 1624.19 & 1743.70 & -0.770 & -0.871 & $8.60(7)$ & $5.91(7)$ \\
\hline $6 s^{2} 6 p\left({ }^{2} P\right) 6 d$ & ${ }^{1} D_{2}^{o}$ & $6 s^{2} 6 p\left({ }^{2} P\right) 7 p$ & ${ }^{3} P_{2}$ & 1483.67 & 1527.50 & -1.328 & -1.018 & $2.85(7)$ & $5.48(7)$ \\
\hline $6 s^{2} 6 p\left({ }^{2} P\right) 6 d$ & ${ }^{3} F_{3}^{o}$ & $6 s^{2} 6 p\left({ }^{2} P\right) 7 p$ & ${ }^{3} P_{2}$ & 977.94 & 1007.90 & -1.842 & -1.834 & $2.06(7)$ & $1.92(7)$ \\
\hline $6 s^{2} 6 p\left({ }^{2} P\right) 6 d$ & ${ }^{3} D_{1}^{o}$ & $6 s^{2} 6 p\left({ }^{2} P\right) 7 p$ & ${ }^{1} S_{0}$ & 934.96 & 967.37 & -2.087 & -2.465 & $6.24(7)$ & $2.44(7)$ \\
\hline
\end{tabular}


TABLE SIII

The wavelengths, $\lambda$, logarithmic weighted oscillator strengths, $\log (g f)$, and transition probabilities, $A_{j i}$, for some E2 and M1 transitions in Rn V. Numbers in brackets represent powers of 10.

\begin{tabular}{|c|c|c|c|c|c|c|c|c|c|}
\hline \multicolumn{4}{|c|}{ Transitions } & \multicolumn{2}{|c|}{$\lambda[\AA]$} & \multicolumn{2}{|c|}{$\log (g f)$} & \multicolumn{2}{|c|}{$A_{j i}\left[\mathrm{~s}^{-1}\right]$} \\
\hline Lower level & Term & Upper level & Term & HFR & MCDF & HFR & MCDF & HFR & MCDF \\
\hline \multicolumn{10}{|c|}{$\mathrm{E} 2$} \\
\hline $6 s^{2} 6 p^{2}$ & ${ }^{3} P_{0}$ & $6 s^{2} 6 p\left({ }^{2} P\right) 7 p$ & ${ }^{3} D_{2}$ & 424.27 & 432.12 & -4.884 & -5.415 & $9.69(4)$ & $2.75(4)$ \\
\hline $6 s^{2} 6 p^{2}$ & ${ }^{3} P_{0}$ & $6 s^{2} 6 p\left({ }^{2} P\right) 7 p$ & ${ }^{3} P_{2}$ & 355.70 & 361.36 & -6.036 & -6.420 & $9.70(3)$ & $3.88(3)$ \\
\hline $6 s^{2} 6 p^{2}$ & ${ }^{3} P_{0}$ & $6 s^{2} 6 p\left({ }^{2} P\right) 7 p$ & ${ }^{3} D_{2}$ & 372.41 & 378.51 & -6.582 & -7.056 & $2.52(3)$ & $0.82(3)$ \\
\hline $6 s^{2} 6 p^{2}$ & ${ }^{3} P_{1}$ & $6 s^{2} 6 p\left({ }^{2} P\right) 7 p$ & ${ }^{3} D_{3}$ & 411.65 & 417.48 & -4.721 & -4.951 & $1.07(5)$ & $0.61(5)$ \\
\hline $6 s^{2} 6 p^{2}$ & ${ }^{3} P_{1}$ & $6 s^{2} 6 p\left({ }^{2} P\right) 7 p$ & ${ }^{3} D_{1}$ & 535.13 & 543.29 & -5.261 & -5.058 & $4.25(4)$ & $6.59(4)$ \\
\hline $6 s^{2} 6 p^{2}$ & ${ }^{3} P_{1}$ & $6 s^{2} 6 p\left({ }^{2} P\right) 7 p$ & ${ }^{3} P_{2}$ & 405.95 & 410.32 & -5.340 & -5.599 & $3.70(4)$ & $2.00(4)$ \\
\hline $6 s^{2} 6 p^{2}$ & ${ }^{3} P_{1}$ & $6 s^{2} 6 p\left({ }^{2} P\right) 7 p$ & ${ }^{3} P_{1}$ & 498.52 & 505.57 & -5.444 & -5.432 & $3.22(4)$ & $3.21(4)$ \\
\hline $6 s^{2} 6 p^{2}$ & ${ }^{3} P_{1}$ & $6 s^{2} 6 p\left({ }^{2} P\right) 7 p$ & ${ }^{3} D_{2}$ & 497.75 & 504.03 & -5.272 & -5.212 & $2.88(4)$ & $3.23(4)$ \\
\hline $6 s^{2} 6 p^{2}$ & ${ }^{3} P_{1}$ & $6 s^{2} 6 p\left({ }^{2} P\right) 7 p$ & ${ }^{3} D_{2}$ & 427.85 & 432.57 & -6.790 & -7.027 & $1.18(3)$ & $0.67(3)$ \\
\hline $6 s^{2} 6 p^{2}$ & ${ }^{3} P_{1}$ & $6 s^{2} 6 p\left({ }^{2} P\right) 7 p$ & ${ }^{3} P_{1}$ & 429.16 & 434.93 & -7.479 & -7.547 & $4.00(2)$ & $3.34(2)$ \\
\hline $6 s^{2} 6 p^{2}$ & ${ }^{1} D_{2}$ & $6 s^{2} 6 p\left({ }^{2} P\right) 7 p$ & ${ }^{3} S_{1}$ & 419.35 & 427.39 & -5.094 & -5.744 & $1.02(5)$ & $0.22(5)$ \\
\hline $6 s^{2} 6 p^{2}$ & ${ }^{1} D_{2}$ & $6 s^{2} 6 p\left({ }^{2} P\right) 7 p$ & ${ }^{1} S_{0}$ & 404.85 & 409.86 & -5.641 & -6.181 & $9.30(4)$ & $2.62(4)$ \\
\hline $6 s^{2} 6 p^{2}$ & ${ }^{1} D_{2}$ & $6 s^{2} 6 p\left({ }^{2} P\right) 7 p$ & ${ }^{3} P_{2}$ & 414.65 & 421.99 & -4.969 & -5.352 & $8.34(4)$ & $3.33(4)$ \\
\hline $6 s^{2} 6 p^{2}$ & ${ }^{1} D_{2}$ & $6 s^{2} 6 p\left({ }^{2} P\right) 7 p$ & ${ }^{3} D_{3}$ & 420.59 & 429.57 & -4.849 & -5.096 & $7.62(4)$ & $4.14(4)$ \\
\hline $6 s^{2} 6 p^{2}$ & ${ }^{1} D_{2}$ & $6 s^{2} 6 p\left({ }^{2} P\right) 7 p$ & ${ }^{3} P_{0}$ & 534.34 & 543.96 & -5.600 & -5.367 & $5.87(4)$ & $9.69(4)$ \\
\hline $6 s^{2} 6 p^{2}$ & ${ }^{1} D_{2}$ & $6 s^{2} 6 p\left({ }^{2} P\right) 7 p$ & ${ }^{3} D_{2}$ & 510.89 & 521.76 & -5.093 & -5.095 & $4.13(4)$ & $3.94(4)$ \\
\hline $6 s^{2} 6 p^{2}$ & ${ }^{1} D_{2}$ & $6 s^{2} 6 p\left({ }^{2} P\right) 7 p$ & ${ }^{3} D_{1}$ & 550.34 & 563.94 & -5.255 & -5.031 & $4.08(4)$ & $6.51(4)$ \\
\hline $6 s^{2} 6 p^{2}$ & ${ }^{1} D_{2}$ & $6 s^{2} 6 p\left({ }^{2} P\right) 7 p$ & ${ }^{3} P_{1}$ & 511.70 & 523.41 & -5.471 & -5.438 & $2.87(4)$ & $2.96(4)$ \\
\hline $6 s^{2} 6 p^{2}$ & ${ }^{1} D_{2}$ & $6 s^{2} 6 p\left({ }^{2} P\right) 7 p$ & ${ }^{3} D_{2}$ & 437.53 & 445.56 & -6.146 & -5.890 & $4.97(3)$ & $8.66(3)$ \\
\hline $6 s^{2} 6 p^{2}$ & ${ }^{3} P_{2}$ & $6 s^{2} 6 p\left({ }^{2} P\right) 7 p$ & ${ }^{1} S_{0}$ & 481.14 & 484.51 & -5.376 & -5.171 & $1.21(5)$ & $1.92(5)$ \\
\hline $6 s^{2} 6 p^{2}$ & ${ }^{3} P_{2}$ & $6 s^{2} 6 p\left({ }^{2} P\right) 7 p$ & ${ }^{3} P_{1}$ & 530.00 & 538.83 & -5.126 & -4.916 & $5.92(4)$ & $9.30(4)$ \\
\hline $6 s^{2} 6 p^{2}$ & ${ }^{3} P_{2}$ & $6 s^{2} 6 p\left({ }^{2} P\right) 7 p$ & ${ }^{3} D_{2}$ & 528.01 & 535.21 & -4.970 & -4.720 & $5.12(4)$ & $8.88(4)$ \\
\hline $6 s^{2} 6 p^{2}$ & ${ }^{3} P_{2}$ & $6 s^{2} 6 p\left({ }^{2} P\right) 7 p$ & ${ }^{3} S_{1}$ & 501.77 & 509.20 & -5.350 & -5.620 & $3.94(4)$ & $2.06(4)$ \\
\hline $6 s^{2} 6 p^{2}$ & ${ }^{3} P_{2}$ & $6 s^{2} 6 p\left({ }^{2} P\right) 7 p$ & ${ }^{3} D_{3}$ & 503.55 & 512.30 & -5.019 & -4.956 & $3.59(4)$ & $4.02(4)$ \\
\hline $6 s^{2} 6 p^{2}$ & ${ }^{3} P_{2}$ & $6 s^{2} 6 p\left({ }^{2} P\right) 7 p$ & ${ }^{3} P_{2}$ & 495.04 & 501.56 & -6.626 & -5.965 & $1.29(3)$ & $5.75(3)$ \\
\hline $6 s^{2} 6 p^{2}$ & ${ }^{3} P_{2}$ & $6 s^{2} 6 p\left({ }^{2} P\right) 7 p$ & ${ }^{3} P_{1}$ & 639.96 & 651.62 & -6.997 & -6.736 & $5.47(2)$ & $9.62(2)$ \\
\hline $6 s^{2} 6 p^{2}$ & ${ }^{3} P_{2}$ & $6 s^{2} 6 p\left({ }^{2} P\right) 7 p$ & ${ }^{3} D_{1}$ & 701.57 & 715.67 & -7.199 & -6.610 & $0.29(3)$ & $1.07(3)$ \\
\hline $6 s^{2} 6 p^{2}$ & ${ }^{3} P_{2}$ & $6 s^{2} 6 p\left({ }^{2} P\right) 7 p$ & ${ }^{3} P_{0}$ & 675.77 & 683.79 & -8.064 & -8.680 & $1.26(2)$ & $0.30(2)$ \\
\hline $6 s^{2} 6 p^{2}$ & ${ }^{3} P_{2}$ & $6 s^{2} 6 p\left({ }^{2} P\right) 7 p$ & ${ }^{3} D_{2}$ & 638.69 & 649.08 & -7.559 & -7.034 & $0.90(2)$ & $2.93(2)$ \\
\hline $6 s^{2} 6 p^{2}$ & ${ }^{1} S_{0}$ & $6 s^{2} 6 p\left({ }^{2} P\right) 7 p$ & ${ }^{3} P_{2}$ & 531.34 & 541.22 & -5.539 & -5.231 & $1.37(4)$ & $2.67(4)$ \\
\hline $6 s^{2} 6 p\left({ }^{2} P\right) 7 p$ & ${ }^{3} D_{1}$ & $6 s^{2} 6 p\left({ }^{2} P\right) 7 p$ & ${ }^{3} D_{2}$ & 2134.36 & 2122.60 & -6.890 & -7.273 & $3.77(1)$ & $1.58(1)$ \\
\hline $6 s^{2} 6 p\left({ }^{2} P\right) 7 p$ & ${ }^{3} D_{1}$ & $6 s^{2} 6 p\left({ }^{2} P\right) 7 p$ & ${ }^{3} P_{1}$ & 7287.53 & 7281.40 & -7.036 & -7.036 & $3.85(0)$ & $3.86(0)$ \\
\hline $6 s^{2} 6 p\left({ }^{2} P\right) 7 p$ & ${ }^{3} D_{1}$ & $6 s^{2} 6 p\left({ }^{2} P\right) 7 p$ & ${ }^{3} D_{2}$ & 7126.42 & 6975.60 & -7.008 & -6.965 & $2.58(0)$ & $2.97(0)$ \\
\hline $6 s^{2} 6 p\left({ }^{2} P\right) 6 d$ & ${ }^{3} F_{2}^{o}$ & $6 s^{2} 6 p\left({ }^{2} P\right) 7 s$ & ${ }^{3} P_{0}^{o}$ & 5223.68 & 5506.90 & -7.349 & -7.413 & $1.09(1)$ & $8.50(0)$ \\
\hline $6 s^{2} 6 p\left({ }^{2} P\right) 6 d$ & ${ }^{3} F_{2}^{o}$ & $6 s^{2} 6 p\left({ }^{2} P\right) 7 s$ & ${ }^{3} P_{1}^{o}$ & 4906.60 & 5232.50 & -7.244 & -7.245 & $5.26(0)$ & $4.62(0)$ \\
\hline $6 s^{2} 6 p\left({ }^{2} P\right) 6 d$ & ${ }^{3} F_{2}^{o}$ & $6 s^{2} 6 p\left({ }^{2} P\right) 7 s$ & ${ }^{3} P_{2}^{o}$ & 1598.74 & 1633.90 & -8.354 & -7.979 & $2.31(0)$ & $5.24(0)$ \\
\hline $6 s^{2} 6 p\left({ }^{2} P\right) 6 d$ & ${ }^{3} F_{3}^{o}$ & $6 s^{2} 6 p\left({ }^{2} P\right) 7 s$ & ${ }^{3} P_{2}^{o}$ & 1941.89 & 2025.60 & -7.540 & -7.204 & $1.02(1)$ & $2.03(1)$ \\
\hline $6 s^{2} 6 p\left({ }^{2} P\right) 6 d$ & ${ }^{3} F_{3}^{o}$ & $6 s^{2} 6 p\left({ }^{2} P\right) 6 d$ & ${ }^{3} D_{3}^{o}$ & 2570.68 & 2542.70 & -7.816 & -7.687 & $2.20(0)$ & $3.03(0)$ \\
\hline $6 s^{2} 6 p\left({ }^{2} P\right) 6 d$ & ${ }^{3} P_{2}^{o}$ & $6 s^{2} 6 p\left({ }^{2} P\right) 6 d$ & ${ }^{1} F_{3}^{o}$ & 1915.77 & 1768.70 & -7.240 & -7.461 & $1.60(1)$ & $1.05(1)$ \\
\hline $6 s^{2} 6 p\left({ }^{2} P\right) 6 d$ & ${ }^{3} P_{2}^{o}$ & $6 s^{2} 6 p\left({ }^{2} P\right) 6 d$ & ${ }^{1} P_{1}^{o}$ & 2046.77 & 1587.80 & -7.690 & -7.555 & $1.08(1)$ & $2.45(1)$ \\
\hline $6 s^{2} 6 p\left({ }^{2} P\right) 6 d$ & ${ }^{3} D_{1}^{o}$ & $6 s^{2} 6 p\left({ }^{2} P\right) 6 d$ & ${ }^{1} P_{1}^{o}$ & 2091.26 & 1639.20 & -7.757 & -8.610 & $8.89(0)$ & $2.03(0)$ \\
\hline $6 s^{2} 6 p\left({ }^{2} P\right) 6 d$ & ${ }^{3} D_{1}^{o}$ & $6 s^{2} 6 p\left({ }^{2} P\right) 7 s$ & ${ }^{1} P_{1}^{o}$ & 1719.02 & 2217.50 & -8.025 & -7.720 & $7.10(0)$ & $8.61(0)$ \\
\hline $6 s^{2} 6 p\left({ }^{2} P\right) 7 s$ & ${ }^{3} P_{1}^{o}$ & $6 s^{2} 6 p\left({ }^{2} P\right) 7 s$ & ${ }^{1} P_{1}^{o}$ & 2000.68 & 2458.50 & -6.782 & -7.463 & $9.17(1)$ & $1.27(1)$ \\
\hline $6 s^{2} 6 p\left({ }^{2} P\right) 7 s$ & ${ }^{3} P_{1}^{o}$ & $6 s^{2} 6 p\left({ }^{2} P\right) 7 s$ & ${ }^{3} P_{2}^{o}$ & 2371.45 & 2375.70 & -6.820 & -6.822 & $3.59(1)$ & $3.56(1)$ \\
\hline $6 s^{2} 6 p\left({ }^{2} P\right) 7 s$ & ${ }^{3} P_{1}^{o}$ & $6 s^{2} 6 p\left({ }^{2} P\right) 6 d$ & ${ }^{1} F_{3}^{o}$ & 2327.24 & 1994.40 & -7.238 & -6.619 & $1.02(1)$ & $5.76(1)$ \\
\hline
\end{tabular}


TABLE SIII (cont.)

\begin{tabular}{|c|c|c|c|c|c|c|c|c|c|}
\hline \multicolumn{4}{|c|}{ Transitions } & \multicolumn{2}{|c|}{$\lambda[\AA]$} & \multicolumn{2}{|c|}{$\log (g f)$} & \multicolumn{2}{|c|}{$A_{j i}\left[\mathrm{~s}^{-1}\right]$} \\
\hline Lower level & Term & Upper level & Term & HFR & MCDF & HFR & MCDF & HFR & MCDF \\
\hline \multicolumn{10}{|c|}{ M1 } \\
\hline $6 s^{2} 6 p\left({ }^{2} P\right) 7 p$ & ${ }^{3} D_{1}$ & $6 s^{2} 6 p\left({ }^{2} P\right) 7 p$ & ${ }^{3} S_{1}$ & 1761.86 & 1765.00 & -7.742 & -7.519 & $1.30(1)$ & $2.16(1)$ \\
\hline $6 s^{2} 6 p\left({ }^{2} P\right) 7 p$ & ${ }^{3} P_{0}$ & $6 s^{2} 6 p\left({ }^{2} P\right) 7 p$ & ${ }^{3} P_{1}$ & 2456.96 & 2177.80 & -5.914 & -6.355 & $4.49(2)$ & $3.73(2)$ \\
\hline $6 s^{2} 6 p\left({ }^{2} P\right) 7 p$ & ${ }^{3} P_{1}$ & $6 s^{2} 6 p\left({ }^{2} P\right) 7 p$ & ${ }^{1} S_{0}$ & 1938.69 & 1889.30 & -6.277 & -6.332 & $9.38(2)$ & $8.70(2)$ \\
\hline $6 s^{2} 6 p\left({ }^{2} P\right) 7 p$ & ${ }^{3} P_{1}$ & $6 s^{2} 6 p\left({ }^{2} P\right) 7 p$ & ${ }^{3} S_{1}$ & 2323.63 & 2329.70 & -5.828 & -5.865 & $6.12(2)$ & $5.59(2)$ \\
\hline $6 s^{2} 6 p\left({ }^{2} P\right) 7 p$ & ${ }^{3} P_{1}$ & $6 s^{2} 6 p\left({ }^{2} P\right) 7 p$ & ${ }^{3} P_{2}$ & 2186.16 & 2177.80 & -5.857 & -5.877 & $3.88(2)$ & $3.73(2)$ \\
\hline $6 s^{2} 6 p\left({ }^{2} P\right) 7 p$ & ${ }^{3} D_{2}$ & $6 s^{2} 6 p\left({ }^{2} P\right) 7 p$ & ${ }^{3} D_{3}$ & 2379.72 & 2431.00 & -5.357 & -5.384 & $7.39(2)$ & $6.66(2)$ \\
\hline $6 s^{2} 6 p\left({ }^{2} P\right) 7 p$ & ${ }^{3} D_{2}$ & $6 s^{2} 6 p\left({ }^{2} P\right) 7 p$ & ${ }^{3} P_{2}$ & 2201.08 & 2206.80 & -5.875 & -5.897 & $3.67(2)$ & $3.47(2)$ \\
\hline $6 s^{2} 6 p\left({ }^{2} P\right) 7 p$ & ${ }^{3} D_{2}$ & $6 s^{2} 6 p\left({ }^{2} P\right) 7 p$ & ${ }^{3} S_{1}$ & 2340.50 & 2362.90 & -6.537 & -6.570 & $1.18(2)$ & $1.07(2)$ \\
\hline $6 s^{2} 6 p\left({ }^{2} P\right) 6 d$ & ${ }^{3} F_{2}^{o}$ & $6 s^{2} 6 p\left({ }^{2} P\right) 6 d$ & ${ }^{3} D_{3}^{o}$ & 2001.88 & 1954.50 & -5.676 & -5.764 & $5.02(2)$ & $4.30(2)$ \\
\hline $6 s^{2} 6 p\left({ }^{2} P\right) 6 d$ & ${ }^{3} F_{2}^{o}$ & $6 s^{2} 6 p\left({ }^{2} P\right) 6 d$ & ${ }^{1} F_{3}^{o}$ & 1578.53 & 1444.00 & -6.134 & -6.150 & $2.81(2)$ & $3.23(2)$ \\
\hline $6 s^{2} 6 p\left({ }^{2} P\right) 6 d$ & ${ }^{3} F_{2}^{o}$ & $6 s^{2} 6 p\left({ }^{2} P\right) 6 d$ & ${ }^{1} D_{2}^{o}$ & 2178.28 & 2193.80 & -6.032 & -6.106 & $2.61(2)$ & $2.17(2)$ \\
\hline $6 s^{2} 6 p\left({ }^{2} P\right) 6 d$ & ${ }^{3} F_{2}^{o}$ & $6 s^{2} 6 p\left({ }^{2} P\right) 6 d$ & ${ }^{3} P_{1}^{o}$ & 1932.77 & 1862.20 & -6.647 & -6.708 & $1.34(2)$ & $1.26(2)$ \\
\hline $6 s^{2} 6 p\left({ }^{2} P\right) 6 d$ & ${ }^{3} F_{2}^{o}$ & $6 s^{2} 6 p\left({ }^{2} P\right) 6 d$ & ${ }^{3} P_{2}^{o}$ & 1876.10 & 1807.70 & -6.480 & -6.470 & $1.25(2)$ & $1.38(2)$ \\
\hline $6 s^{2} 6 p\left({ }^{2} P\right) 6 d$ & ${ }^{3} F_{2}^{o}$ & $6 s^{2} 6 p\left({ }^{2} P\right) 6 d$ & ${ }^{3} F_{3}^{o}$ & 9047.50 & 8448.60 & -5.757 & -5.711 & $2.04(1)$ & $2.59(1)$ \\
\hline $6 s^{2} 6 p\left({ }^{2} P\right) 6 d$ & ${ }^{3} F_{2}^{o}$ & $6 s^{2} 6 p\left({ }^{2} P\right) 7 s$ & ${ }^{1} P_{1}^{o}$ & 1421.19 & 1672.60 & -8.251 & -8.862 & $6.13(0)$ & $1.09(0)$ \\
\hline $6 s^{2} 6 p\left({ }^{2} P\right) 6 d$ & ${ }^{3} F_{3}^{o}$ & $6 s^{2} 6 p\left({ }^{2} P\right) 6 d$ & ${ }^{3} F_{4}^{o}$ & 2876.48 & 3037.50 & -5.204 & -5.215 & $5.60(2)$ & $4.90(2)$ \\
\hline $6 s^{2} 6 p\left({ }^{2} P\right) 6 d$ & ${ }^{3} F_{3}^{o}$ & $6 s^{2} 6 p\left({ }^{2} P\right) 6 d$ & ${ }^{1} F_{3}^{o}$ & 1912.14 & 1741.70 & -5.943 & -6.028 & $2.97(2)$ & $2.94(2)$ \\
\hline $6 s^{2} 6 p\left({ }^{2} P\right) 6 d$ & ${ }^{3} F_{3}^{o}$ & $6 s^{2} 6 p\left({ }^{2} P\right) 6 d$ & ${ }^{3} D_{3}^{o}$ & 2570.68 & 2542.70 & -6.153 & -6.150 & $1.04(2)$ & $1.04(2)$ \\
\hline $6 s^{2} 6 p\left({ }^{2} P\right) 6 d$ & ${ }^{3} F_{3}^{o}$ & $6 s^{2} 6 p\left({ }^{2} P\right) 6 d$ & ${ }^{3} P_{2}^{o}$ & 2366.90 & 2299.80 & -6.400 & -6.487 & $9.49(1)$ & $8.22(1)$ \\
\hline $6 s^{2} 6 p\left({ }^{2} P\right) 6 d$ & ${ }^{3} P_{2}^{o}$ & $6 s^{2} 6 p\left({ }^{2} P\right) 7 s$ & ${ }^{1} P_{1}^{o}$ & 1688.85 & 2124.30 & -6.263 & -6.404 & $4.25(2)$ & $1.94(2)$ \\
\hline $6 s^{2} 6 p\left({ }^{2} P\right) 6 d$ & ${ }^{3} P_{2}^{o}$ & $6 s^{2} 6 p\left({ }^{2} P\right) 6 d$ & ${ }^{1} P_{1}^{o}$ & 2046.77 & 1587.80 & -6.129 & -6.137 & $3.94(2)$ & $6.43(2)$ \\
\hline $6 s^{2} 6 p\left({ }^{2} P\right) 6 d$ & ${ }^{3} P_{2}^{o}$ & $6 s^{2} 6 p\left({ }^{2} P\right) 6 d$ & ${ }^{3} P_{2}^{o}$ & 2372.46 & 2347.10 & -5.797 & -5.849 & $3.78(2)$ & $3.43(2)$ \\
\hline $6 s^{2} 6 p\left({ }^{2} P\right) 6 d$ & ${ }^{3} P_{2}^{o}$ & $6 s^{2} 6 p\left({ }^{2} P\right) 6 d$ & ${ }^{1} F_{3}^{o}$ & 1915.77 & 1768.70 & -6.020 & -6.085 & $2.48(2)$ & $2.50(2)$ \\
\hline $6 s^{2} 6 p\left({ }^{2} P\right) 6 d$ & ${ }^{3} P_{2}^{o}$ & $6 s^{2} 6 p\left({ }^{2} P\right) 6 d$ & ${ }^{1} D_{2}^{o}$ & 2877.21 & 3042.20 & -6.100 & -6.046 & $1.28(2)$ & $1.30(2)$ \\
\hline $6 s^{2} 6 p\left({ }^{2} P\right) 6 d$ & ${ }^{3} P_{2}^{o}$ & $6 s^{2} 6 p\left({ }^{2} P\right) 6 d$ & ${ }^{3} D_{3}^{o}$ & 2577.24 & 2600.70 & -6.209 & -6.189 & $8.86(1)$ & $9.11(1)$ \\
\hline $6 s^{2} 6 p\left({ }^{2} P\right) 6 d$ & ${ }^{3} P_{2}^{o}$ & $6 s^{2} 6 p\left({ }^{2} P\right) 6 d$ & ${ }^{3} P_{1}^{o}$ & 2463.82 & 2439.80 & -7.104 & -7.041 & $2.88(1)$ & $3.40(1)$ \\
\hline $6 s^{2} 6 p\left({ }^{2} P\right) 6 d$ & ${ }^{3} D_{1}^{o}$ & $6 s^{2} 6 p\left({ }^{2} P\right) 6 d$ & ${ }^{3} P_{0}^{o}$ & 2540.79 & 2598.40 & -6.242 & -6.254 & $5.92(2)$ & $5.50(2)$ \\
\hline $6 s^{2} 6 p\left({ }^{2} P\right) 6 d$ & ${ }^{3} D_{1}^{o}$ & $6 s^{2} 6 p\left({ }^{2} P\right) 6 d$ & ${ }^{3} P_{1}^{o}$ & 2528.56 & 2563.40 & -5.872 & -5.877 & $4.67(2)$ & $4.49(2)$ \\
\hline $6 s^{2} 6 p\left({ }^{2} P\right) 6 d$ & ${ }^{3} D_{1}^{o}$ & $6 s^{2} 6 p\left({ }^{2} P\right) 6 d$ & ${ }^{1} D_{2}^{o}$ & 2965.89 & 3237.00 & -5.926 & -5.939 & $1.80(2)$ & $1.46(2)$ \\
\hline $6 s^{2} 6 p\left({ }^{2} P\right) 6 d$ & ${ }^{3} D_{1}^{o}$ & $6 s^{2} 6 p\left({ }^{2} P\right) 6 d$ & ${ }^{3} P_{2}^{o}$ & 2432.44 & 2461.30 & -6.361 & -6.308 & $0.98(2)$ & $1.08(2)$ \\
\hline $6 s^{2} 6 p\left({ }^{2} P\right) 6 d$ & ${ }^{3} D_{1}^{o}$ & $6 s^{2} 6 p\left({ }^{2} P\right) 7 s$ & ${ }^{3} P_{2}^{o}$ & 1985.78 & 2149.90 & -7.024 & -7.333 & $3.20(1)$ & $1.34(1)$ \\
\hline $6 s^{2} 6 p\left({ }^{2} P\right) 6 d$ & ${ }^{3} D_{3}^{o}$ & $6 s^{2} 6 p\left({ }^{2} P\right) 6 d$ & ${ }^{1} F_{3}^{o}$ & 7464.26 & 5528.80 & -6.231 & -6.148 & $1.00(1)$ & $2.22(1)$ \\
\hline $6 s^{2} 6 p\left({ }^{2} P\right) 7 s$ & ${ }^{3} P_{0}^{o}$ & $6 s^{2} 6 p\left({ }^{2} P\right) 7 s$ & ${ }^{1} P_{1}^{o}$ & 1952.35 & 2402.20 & -6.297 & -6.111 & $2.94(2)$ & $2.99(2)$ \\
\hline $6 s^{2} 6 p\left({ }^{2} P\right) 7 s$ & ${ }^{3} P_{0}^{o}$ & $6 s^{2} 6 p\left({ }^{2} P\right) 6 d$ & ${ }^{1} P_{1}^{o}$ & 2447.05 & 1738.10 & -6.219 & -6.539 & $2.24(2)$ & $2.13(2)$ \\
\hline $6 s^{2} 6 p\left({ }^{2} P\right) 7 s$ & ${ }^{3} P_{1}^{o}$ & $6 s^{2} 6 p\left({ }^{2} P\right) 7 s$ & ${ }^{3} P_{2}^{o}$ & 2371.45 & 2375.70 & -5.541 & -5.558 & $6.83(2)$ & $6.55(2)$ \\
\hline $6 s^{2} 6 p\left({ }^{2} P\right) 7 s$ & ${ }^{3} P_{1}^{o}$ & $6 s^{2} 6 p\left({ }^{2} P\right) 7 s$ & ${ }^{1} P_{1}^{o}$ & 2000.68 & 2458.50 & -6.687 & -6.389 & $1.14(2)$ & $1.50(2)$ \\
\hline $6 s^{2} 6 p\left({ }^{2} P\right) 7 s$ & ${ }^{3} P_{1}^{o}$ & $6 s^{2} 6 p\left({ }^{2} P\right) 6 d$ & ${ }^{1} P_{1}^{o}$ & 2523.44 & 1767.30 & -6.490 & -6.928 & $1.13(2)$ & $8.39(1)$ \\
\hline $6 s^{2} 6 p\left({ }^{2} P\right) 7 s$ & ${ }^{3} P_{1}^{o}$ & $6 s^{2} 6 p\left({ }^{2} P\right) 6 d$ & ${ }^{3} P_{1}^{o}$ & 3188.92 & 2891.10 & -7.001 & -7.214 & $2.18(1)$ & $1.63(1)$ \\
\hline $6 s^{2} 6 p\left({ }^{2} P\right) 7 s$ & ${ }^{3} P_{1}^{o}$ & $6 s^{2} 6 p\left({ }^{2} P\right) 6 d$ & ${ }^{3} P_{0}^{o}$ & 3208.40 & 2935.60 & -7.577 & -7.828 & $1.71(1)$ & $1.15(1)$ \\
\hline
\end{tabular}

